\title{
Computing the Interleaving Distance is NP-Hard
}

\author{
Håvard Bakke Bjerkevik ${ }^{1} \cdot$ Magnus Bakke Botnan ${ }^{2} \cdot$ Michael Kerber $^{3}$
}

Received: 22 November 2018 / Revised: 8 August 2019 / Accepted: 10 September 2019 /

Published online: 11 November 2019

(c) The Author(s) 2019

\begin{abstract}
We show that computing the interleaving distance between two multi-graded persistence modules is NP-hard. More precisely, we show that deciding whether two modules are 1-interleaved is NP-complete, already for bigraded, interval decomposable modules. Our proof is based on previous work showing that a constrained matrix invertibility problem can be reduced to the interleaving distance computation of a special type of persistence modules. We show that this matrix invertibility problem is NP-complete. We also give a slight improvement in the above reduction, showing that also the approximation of the interleaving distance is NP-hard for any approximation factor smaller than 3. Additionally, we obtain corresponding hardness results for the case that the modules are indecomposable, and in the setting of one-sided stability. Furthermore, we show that checking for injections (resp. surjections) between persistence modules is NP-hard. In conjunction with earlier results from computational algebra this gives a complete characterization of the computational complexity of onesided stability. Lastly, we show that it is in general NP-hard to approximate distances induced by noise systems within a factor of 2 .
\end{abstract}

Keywords NP-hardness · Persistent homology $\cdot$ Interleavings $\cdot$ Matrix completion problems

Mathematics Subject Classification $15 \mathrm{~A} 83 \cdot 55 \mathrm{U} 99$

Communicated by Herbert Edelsbrunner.

$\bowtie$ Magnus Bakke Botnan

m.b.botnan@vu.nl

Håvard Bakke Bjerkevik

havard.bjerkevik@ntnu.no

Michael Kerber

kerber@tugraz.at

1 Institutt for matematiske fag, NTNU, 7491 Trondheim, Norway

2 Department of Mathematics, Vrije Universiteit Amsterdam, 1081 HV Amsterdam, The Netherlands

3 Institut für Geometrie, TU Graz, 8010 Graz, Austria 


\section{Introduction}

\subsection{Motivation and Problem Statement}

A persistence module $M$ over $\mathbb{R}^{d}$ is a collection of vector spaces $\left\{M_{p}\right\}_{p \in \mathbb{R}^{d}}$ and linear maps $M_{p \rightarrow q}: M_{p} \rightarrow M_{q}$ whenever $p \leq q$, with the property that $M_{p \rightarrow p}$ is the identity map and the linear maps are composable in the obvious way. For $d=1$, we will talk about single-parameter persistence, and for $d \geq 2$, we will use the term multi-parameter persistence.

Persistence, particularly in its single-parameter version, has recently gained a lot of attention in applied fields, because one of its instantiations is persistent homology, which studies the evolution of homology groups when varying a real scale parameter. The observation that topological features in real data sets carry important information to analyze and reason about the contained data has given rise to the term topological data analysis (TDA) for this research field, with various connections to application areas, e.g., [2,9,16-18].

A recurring task in TDA is the comparison of two persistence modules. The natural notion in terms of algebra is by interleavings of two persistence modules: given two persistence modules $M$ and $N$ as above and some $\epsilon>0$, an $\epsilon$-interleaving is the assignment of maps $\phi_{p}: M_{p} \rightarrow N_{p+\epsilon}$ and $\psi_{p}: N_{p} \rightarrow M_{p+\epsilon}$ which commute with each other and the internal maps of $M$ and $N$. The interleaving distance is then just the infimum over all $\epsilon$ for which an interleaving exists.

A desirable property for any distance on persistence modules is stability, meaning informally that a small change in the input data set should only lead to a small distortion of the distance. At the same time, we aim for a sensitive measure, meaning that the distance between modules should be generally as large as possible without violating stability. As an extreme example, the distance measure that assigns 0 to all pairs of modules is maximally stable, but also maximally insensitive. Lesnick [14] proved that among all stable distances for single- or multi-parameter persistence, the interleaving distance is the most sensitive one over prime fields. This makes the interleaving distance an interesting measure to be used in applications and raises the question of how costly it is to compute the distance [14, Sec. 1.3 and 7]. Of course, for the sake of computation, a suitable finiteness condition must be imposed on the modules to ensure that they can be represented in finite form; we postpone the discussion to Sect. 3, and simply call such modules of finite type.

The complexity of computing the interleaving distance is well understood for the single-parameter case. The isometry theorem $[8,14]$ states the equivalence of the interleaving distance and the bottleneck distance, which is defined in terms of the persistence diagrams of the persistence modules and can be reduced to the computation of a min cost bottleneck matching in a complete bipartite graph [11]. That matching, in turn, can be computed in $O\left(n^{1.5} \log n\right)$ time, and efficient implementations have been developed recently [13].

The described strategy, however, fails in the multi-parameter case, simply because the two distances do not match for more than one parameter: even if the multi-parameter persistence module admits a decomposition into intervals (which are "nice" indecom-

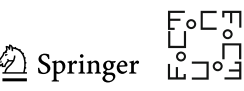


posable elements, see Sect. 3), it has been proved that the interleaving distance and the multi-parameter extension of the bottleneck distance are arbitrarily far from each other [5, Example 9.1]. Another example where the interleaving and bottleneck distances differ is given in [3, Example 4.2]; moreover, in this example the pair of persistence modules has the property that potential interleavings can be written on a particular matrix form, later formalized by the introduction of CI problems in [4]. A consequence is that the strategy of computing interleaving distance by computing the bottleneck distance fails also in this special case.

\subsection{Our Contributions}

We show that, for $d=2$, the computation of the interleaving distance of two persistence modules of finite type is NP-hard, even if the modules are assumed to be decomposable into intervals. In [4], it is proved that the problem is CI-hard, where CI is a combinatorial problem related to the invertibility of a matrix with a prescribed set of zero elements. This is done by associating a pair of modules to each CI problem such that the modules are 1-interleaved if and only if the CI problem has a solution. We "finish" this proof by showing that CI is NP-complete, hence proving the main result. The hardness result on $\mathrm{CI}$ is independent of all topological concepts required for the rest of the paper and potentially of independent interest in other algorithmic areas.

Moreover, we slightly improve the reduction from [4] that asserts the CI-hardness of the interleaving distance, showing that also obtaining a $(3-\epsilon)$-approximation of the interleaving distance is NP-hard to obtain for every $\epsilon>0$. This result follows from the fact that our improved construction takes an instance of a CI problem and returns a pair of persistence modules which are 1-interleaved if the instance has a solution and are 3-interleaved if no solution exists. We mention that for rectangle decomposable modules in $d=2$, a subclass of interval decomposable modules, it is known that the bottleneck distance 3-approximates the interleaving distance [3, Theorem 3.2], and can be computed in polynomial time. While this result does not directly extend to all interval decomposable modules, it gives reason to hope that a 3-approximation of the interleaving distance exists for a larger class of modules.

We also extend our hardness result to related problems: we show that it is NPcomplete to compute the interleaving distance of two indecomposable persistence modules (for $d=2$ ). We obtain this result by "stitching" together the interval decomposables from our main result into two indecomposable modules without affecting their interleaving distance. We remark that the restriction of computing the interleaving distance of indecomposable interval modules has recently been shown to be in $\mathrm{P}$ [10].

Bauer and Lesnick [1] showed that the existence of an interleaving pair, for modules indexed over $\mathbb{R}$, is equivalent to the existence of a single morphism with kernel and cokernel of a corresponding "size". While the equivalence does not hold in general, the two concepts are still closely related for $d>1$. Using this, we obtain as a corollary to 
the aforementioned results that it is in general NP-complete to decide if there exists a morphism whose kernel and cokernel have size bounded by a given parameter. We also show that it is NP-complete to decide if there exists a surjection (dually, an injection) from one persistence module to another. Together with the result of [6], this gives a complete characterization of the computational complexity of "one-sided stability". Furthermore, we remark that this gives an alternative proof of the fact that checking for injections (resp. surjections) between modules over a finite-dimensional algebra (over a finite field) is NP-hard. This was first shown in [12, Theorem 1.2] (for arbitrary fields). The paper concludes with a result showing that it is in general NP-hard to approximate distances induced by noise systems (as introduced by Scolamiero et al. [19]) within a factor of 2 .

\subsection{Outline}

We begin with the hardness proof for CI in Sect. 2. In Sect. 3, we discuss the representation-theoretic concepts needed in the paper. In Sect. 4, we describe our improved reduction scheme from interleaving distance to CI. In Sect. 5, we prove the hardness for indecomposable modules. In Sect. 6, we prove our hardness result for one-sided stability. A result closely related to one-sided stability can be found in Sect. 7 where we discuss a particular distance induced by a noise system. We conclude in Sect. 8.

\section{The CI Problem}

Throughout the paper, we set $\mathbb{F}$ to be any finite field with a constant number of elements. We write $\mathbb{F}^{n \times n}$ for the set of $n \times n$-matrices over $\mathbb{F}$, and $P_{i j} \in \mathbb{F}$ for the entry of $P$ at the position at row $i$ and column $j$. We write $I_{n}$ for the $n \times n$-unit matrix. The constrained invertibility problem asks for a solution of the equation $A B=I_{n}$, when certain entries of $A$ and of $B$ are constrained to be zero. Formally, using the notation $[n]:=\{1, \ldots, n\}$, we define the language

$$
\begin{aligned}
\mathrm{CI}:= & \left\{(n, P, Q) \mid P \subseteq[n] \times[n] \wedge Q \subseteq[n] \times[n] \wedge \exists A, B \in \mathbb{F}^{n \times n}:\right. \\
& \left.\left(\forall(i, j) \in P: A_{i, j}=0 \wedge \forall(i, j) \in Q: B_{i, j}=0 \wedge A B=I_{n}\right)\right\} .
\end{aligned}
$$

We can write CI-instances in a more visual form, for instance writing

$$
\left(\begin{array}{ccc}
* & * & * \\
* & 0 & * \\
* & * & 0
\end{array}\right)\left(\begin{array}{lll}
* & * & * \\
* & * & 0 \\
* & 0 & *
\end{array}\right)=I_{3}
$$

instead of $(3,\{(2,2),(3,3)\},\{(2,3),(3,2)\})$. Indeed, the CI problem asks whether in the above matrices, we can fill the $*$-entries with field elements to satisfy the equation. 
In the above example, this is indeed possible, for instance by choosing

$$
A=\left(\begin{array}{lll}
1 & 1 & 1 \\
1 & \mathbf{0} & 1 \\
1 & 1 & \mathbf{0}
\end{array}\right) \quad B=\left(\begin{array}{ccc}
-1 & 1 & 1 \\
1 & -1 & \mathbf{0} \\
1 & \mathbf{0} & -1
\end{array}\right)
$$

We sometimes also call $A$ and $B$ a satisfying assignment. In contrast, the instance

$$
\left(\begin{array}{lll}
0 & * & 0 \\
* & * & * \\
* & * & *
\end{array}\right)\left(\begin{array}{lll}
* & * & * \\
0 & * & * \\
* & * & *
\end{array}\right)=I_{3}
$$

has no solution, because the $(1,1)$ entry of the product on the left is always 0 , no matter what values are chosen. Note that the existence of a solution also depends on the characteristic of the base field. For an example, see Chapter 4, page 13 in [4].

The CI problem is of interest to us, because we will see in Sect. 4 that CI reduces to the problem of computing the interleaving distance, that is, a polynomial time algorithm for computing the interleaving distance will allow us to decide whether a triple $(n, P, Q)$ is in $C I$, also in polynomial time. Although the definition of CI is rather elementary and appears to be useful in different contexts, we are not aware of any previous work studying this problem (apart from [4]).

It is clear that $C I$ is in NP because a valid choice of the matrices $A$ and $B$ can be checked in polynomial time. We want to show that $C I$ is NP-hard as well. It will be convenient to do so in two steps. First, we define a slightly more general problem, called generalized constrained invertibility $(G C I)$, and show that GCI reduces to CI. Then, we proceed by showing that 3SAT reduces to GCI, proving the NP-hardness of CI.

\subsection{Generalized Constrained Invertibility}

We generalize from the above problem in two ways: first, instead of square matrices, we allow that $A \in \mathbb{F}^{n \times m}$ and $B \in \mathbb{F}^{m \times n}$ (where $m$ is an additional input). Second, instead of forcing $A B=I_{n}$, we only require that $A B$ coincides with $I_{n}$ in a fixed subset of entries over $[n] \times[n]$. Formally, we define

$$
\begin{aligned}
G C I:= & \{(n, m, P, Q, R) \mid P \subseteq[n] \times[m] \wedge Q \subseteq[m] \times[n] \wedge R \subseteq[n] \\
& \times[n] \wedge \exists A \in \mathbb{F}^{n \times m}, B \in \mathbb{F}^{m \times n}: \\
& \left(\forall(i, j) \in P: A_{i, j}=0 \wedge \forall(i, j) \in Q: B_{i, j}\right. \\
= & \left.\left.0 \wedge \forall(i, j) \in R:(A B)_{i, j}=\left(I_{n}\right)_{i, j}\right)\right\} .
\end{aligned}
$$

Again, we use the following notation

$$
\left(\begin{array}{ccc}
* & * & * \\
0 & 0 & 0
\end{array}\right)\left(\begin{array}{ll}
* & 0 \\
0 & * \\
0 & 0
\end{array}\right)=\left(\begin{array}{ll}
1 & 0 \\
* & *
\end{array}\right)
$$


for the GCI-instance $(2,3,\{(2,1),(2,2),(2,3)\},\{(1,2),(2,1),(3,1),(3,2)\},\{(1,1)$, $(1,2)\})$. This instance is indeed in GCI, as for instance,

$$
\left(\begin{array}{lll}
1 & 0 & 0 \\
\mathbf{0} & \mathbf{0} & \mathbf{0}
\end{array}\right)\left(\begin{array}{ll}
1 & \mathbf{0} \\
\mathbf{0} & 1 \\
\mathbf{0} & \mathbf{0}
\end{array}\right)=\left(\begin{array}{ll}
\mathbf{1} & \mathbf{0} \\
0 & 0
\end{array}\right)
$$

GCI is indeed generalizing CI, as we can encode any CI-instance by setting $m=n$ and $R=[n] \times[n]$. Hence, CI trivially reduces to GCI. We show, however, that also the converse is true, meaning that the problems are computationally equivalent. We will need the following lemma which follows from linear algebra:

Lemma 1 Let $M \in \mathbb{F}^{n \times m}, N \in \mathbb{F}^{m \times n}$ with $m>n$ such that $M N=I_{n}$. Then there exist matrices $M^{\prime} \in \mathbb{F}^{(m-n) \times m}, N^{\prime} \in \mathbb{F}^{m \times(m-n)}$ such that

$$
\left[\begin{array}{c}
M \\
M^{\prime}
\end{array}\right]\left[\begin{array}{ll}
N & N^{\prime}
\end{array}\right]=I_{m}
$$

Proof Pick $M^{\prime \prime} \in \mathbb{F}^{(m-n) \times m}$ so that $\left[\begin{array}{c}M \\ M^{\prime \prime}\end{array}\right]$ has full rank. This is possible, as the row vectors of $M$ are linearly independent, so we can pick the rows in $M^{\prime \prime}$ iteratively such that they are linearly independent of each other and those in $M$. Let $M^{\prime}=$ $M^{\prime \prime}-M^{\prime \prime} N M$, which gives

$$
M^{\prime} N=\left(M^{\prime \prime}-M^{\prime \prime} N M\right) N=M^{\prime \prime} N-M^{\prime \prime} N I_{n}=0_{(m-n) \times n} .
$$

Since

$$
\left[\begin{array}{c}
M \\
M^{\prime \prime}
\end{array}\right]=\left[\begin{array}{cc}
I_{n} & 0_{n \times(m-n)} \\
M^{\prime \prime} N & I_{m-n}
\end{array}\right]\left[\begin{array}{c}
M \\
M^{\prime}
\end{array}\right]
$$

$\left[\begin{array}{l}M \\ M^{\prime}\end{array}\right]$ also has full rank, which means that it has an inverse. Let $N^{\prime}$ be the last $m-n$ columns of this inverse matrix. We get

$$
\left[\begin{array}{c}
M \\
M^{\prime}
\end{array}\right]\left[\begin{array}{ll}
N & N^{\prime}
\end{array}\right]=\left[\begin{array}{cc}
I_{n} & 0_{n \times(m-n)} \\
0_{(m-n) \times n} & I_{m-n}
\end{array}\right]=I_{m} .
$$

Lemma 2 GCI is polynomial time reducible to $C I$.

Proof Fix a GCI-instance $(n, m, P, Q, R)$. We have to define a polynomial time algorithm to compute a CI-instance $\left(n^{\prime}, P^{\prime}, Q^{\prime}\right)$ such that

$$
(n, m, P, Q, R) \in G C I \Leftrightarrow\left(n^{\prime}, P^{\prime}, Q^{\prime}\right) \in C I .
$$

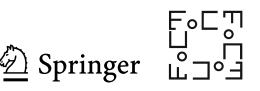


Write the GCI-instance as $A B=C$, where $A$ and $B$ are matrices with 0 and $*$ entries (of dimensions $n \times m$ and $m \times n$, respectively), and $C$ is an $n \times n$-matrix with 1 or $*$ entries on the diagonal, and 0 or $*$ entries away from the diagonal (as in the example above).

Define the matrix $I_{n}^{*}$ as the matrix with 0 away from the diagonal and $*$ on the diagonal. Moreover, let $\bar{C}$ denote the matrix $C$ with all 1 -entries replaced by 0 -entries. Now, consider the GCI-instance

$$
\left[\begin{array}{ll}
A & I_{n}^{*}
\end{array}\right]\left[\begin{array}{c}
B \\
\bar{C}
\end{array}\right]=I_{n}
$$

which can be formally written as $\left(n, n+m, P^{\prime}, Q^{\prime},[n] \times[n]\right)$ for some choices $P^{\prime} \supseteq P$, $Q^{\prime} \supseteq Q$.

We claim that the original instance is in GCI if and only if the extended instance is in GCI. First, assume that $A B=C$ has a solution (that is, an assignment of field elements to $*$ entries that satisfies the equation). Then, we pick all diagonal entries in $I_{n}^{*}$ as 1 , so that the matrix becomes $I_{n}$. Also, we pick $\bar{C}$ to be $I_{n}-A B$; this is indeed possible, as an entry in $\bar{C}$ is fixed only if the corresponding positions of $I_{n}$ and $A B$ coincide. With these choices, we have that

$$
\left[\begin{array}{ll}
A & I_{n}^{*}
\end{array}\right]\left[\begin{array}{c}
B \\
\bar{C}
\end{array}\right]=A B+I_{n}\left(I_{n}-A B\right)=I_{n},
$$

as required.

Conversely, if there is a solution for the extended instance, write $X$ for the assignment of $I_{n}^{*}$ and $Y$ for the assignment of $\bar{C}$. Then $A B+X Y=I_{n}$. Now fix any index $(i, j) \in R$ and consider the equation in that entry. By construction $Y_{i, j}=0$, and multiplication by the diagonal matrix $X$ does not change this property. It follows that $(A B)_{(i, j)}=\left(I_{n}\right)_{i, j}$, which means that $A B=C$ has a solution. Hence, the two instances are indeed equivalent.

To finish the proof, we observe that (1) is in GCI if and only if

$$
\left[\begin{array}{cc}
A & I_{n}^{*} \\
*_{m \times m} & *_{m \times n}
\end{array}\right]\left[\begin{array}{cc}
B & *_{m \times m} \\
\bar{C} & *_{n \times m}
\end{array}\right]=I_{n+m}
$$

is in GCI, where $*_{a \times b}$ is simply the $a \times b$ matrix only containing $*$ entries. Formally written, this instance corresponds to $\left(n+m, n+m, P^{\prime}, Q^{\prime},[n+m] \times[n+m]\right)$. To see the equivalence, if (1) is in GCI, Lemma 1 asserts that there are indeed choices for the $*$-matrices to solve (2) as well. In the opposite direction, a satisfying assignment of the involved matrices in (2) also yields a valid solution for (1) when restricted to the upper $n$ rows and left $n$ columns, respectively.

Combining everything, we see that $(n, m, P, Q, R)$ is in GCI if and only if ( $n+$ $\left.m, n+m, P^{\prime}, Q^{\prime},[n+m] \times[n+m]\right)$ is in GCI. The latter, however, is equivalent to the CI-instance $\left(n+m, P^{\prime}, Q^{\prime}\right)$. The conversion can clearly be performed in polynomial time, and the statement follows. 


\subsection{Hardness of $\mathbf{G C l}$}

We describe now how an algorithm for how deciding GCI can be used to decide satisfiability of 3SAT formulas. Let $\phi$ be a 3CNF formula with $n$ variables and $m$ clauses. We construct a GCI-instance that is satisfiable if and only if $\phi$ is satisfiable.

In what follows, we will often label some $*$ entries in matrices with variables when we want to talk about the possible assignments of the corresponding entries.

The first step is to build a "gadget" that allows us to encode the truth value of a variable in the matrix. Consider the instance

$$
\left(\begin{array}{ccc}
* & 0 & * \\
0 & * & *
\end{array}\right)\left(\begin{array}{ll}
x & 0 \\
0 & y \\
* & *
\end{array}\right)=I_{2}
$$

In any solution to this equation, not both $x$ and $y$ can be zero because otherwise, the right matrix would have rank at most 1 . Furthermore, when extending the instance by one row/column

$$
\left(\begin{array}{ccc}
a & b & 0 \\
\hdashline * & 0 & * \\
0 & * & *
\end{array}\right)\left(\begin{array}{ccc}
0 & x & 0 \\
0 & 0 & y \\
0 & * & *
\end{array}\right)=\left(\begin{array}{ccc}
* & 0 & 0 \\
0 & 1 & 0 \\
0 & 0 & 1
\end{array}\right)
$$

we see that both $a x=0$ and $b y=0$ must hold, which is then only possible if at least one entry $a$ or $b$ is equal to 0 . In fact, there is a solution with $a \neq 0$, and a solution with $b \neq 0$, for instance

$$
\begin{aligned}
& \left(\begin{array}{lll}
1 & 0 & \mathbf{0} \\
0 & \mathbf{0} & 1 \\
\mathbf{0} & 1 & 0
\end{array}\right)\left(\begin{array}{lll}
\mathbf{0} & 0 & \mathbf{0} \\
\mathbf{0} & \mathbf{0} & 1 \\
\mathbf{0} & 1 & 0
\end{array}\right)=\left(\begin{array}{lll}
0 & \mathbf{0} & \mathbf{0} \\
\mathbf{0} & \mathbf{1} & \mathbf{0} \\
\mathbf{0} & \mathbf{0} & \mathbf{1}
\end{array}\right), \\
& \left(\begin{array}{lll}
0 & 1 & \mathbf{0} \\
1 & \mathbf{0} & 0 \\
\mathbf{0} & 0 & 1
\end{array}\right)\left(\begin{array}{lll}
\mathbf{0} & 1 & \mathbf{0} \\
\mathbf{0} & \mathbf{0} & 0 \\
\mathbf{0} & 0 & 1
\end{array}\right)=\left(\begin{array}{lll}
0 & \mathbf{0} & \mathbf{0} \\
\mathbf{0} & \mathbf{1} & \mathbf{0} \\
\mathbf{0} & \mathbf{0} & \mathbf{1}
\end{array}\right) .
\end{aligned}
$$

The intuition is that for a variable $x_{i}$ appearing in $\phi$, we interpret $x_{i}$ to be true if $a \neq 0$, and to be false if $b \neq 0$. We build such a gadget for each variable. A crucial observation is that we can do so with all variable entries placed in the same row. This works essentially by concatenating the variable gadgets, in a block-like fashion. We show the construction for three variables as an example.

$$
\left(\begin{array}{cccccccccc}
a_{0} & b_{0} & 0 & a_{1} & b_{1} & 0 & a_{2} & b_{2} & 0 & * \\
* & 0 & * & 0 & 0 & 0 & 0 & 0 & 0 & 0 \\
0 & * & * & 0 & 0 & 0 & 0 & 0 & 0 & 0 \\
0 & 0 & 0 & * & 0 & * & 0 & 0 & 0 & 0 \\
0 & 0 & 0 & 0 & * & * & 0 & 0 & 0 & 0 \\
0 & 0 & 0 & 0 & 0 & 0 & * & 0 & * & 0 \\
0 & 0 & 0 & 0 & 0 & 0 & 0 & * & * & 0
\end{array}\right)\left(\begin{array}{ccccccc}
0 & * & 0 & 0 & 0 & 0 & 0 \\
0 & 0 & * & 0 & 0 & 0 & 0 \\
0 & * & * & 0 & 0 & 0 & 0 \\
0 & 0 & 0 & * & 0 & 0 & 0 \\
0 & 0 & 0 & 0 & * & 0 & 0 \\
0 & 0 & 0 & * & * & 0 & 0 \\
0 & 0 & 0 & 0 & 0 & * & 0 \\
0 & 0 & 0 & 0 & 0 & 0 & * \\
0 & 0 & 0 & 0 & 0 & * & * \\
* & 0 & 0 & 0 & 0 & 0 & 0
\end{array}\right)=I_{7}
$$


where we introduced an additional column at the end of the left matrix and an additional row at the end of the second matrix. Firstly, this allows us to satisfy the entire $I_{7}$ on the right-hand side; moreover, it will be useful when extending the construction to clauses. It is straightforward to generalize this construction to an arbitrary number of variables. We arrive at the following intermediate result.

Lemma 3 For any $n \geq 1$, there exists a GCI-instance $A^{\prime} B^{\prime}=I_{2 n+1}$ with $A^{\prime}$ having $3 n+1$ columns, such that in each solution for the problem, $A_{1,3 n+1}^{\prime}$ is not zero, and for each $k=0, \ldots, n-1$, the entries $A_{1,3 k+1}^{\prime}$ and $A_{1,3 k+2}^{\prime}$ are not both non-zero. Moreover, for any choice of $v_{1}, \ldots, v_{n} \in\{1,2\}$, there exists a solution of the instance in which $A_{1,3 k+v_{i}}^{\prime} \neq 0$ for all $k=0, \ldots, n-1$.

Next, we extend the instance from Lemma 3 with respect to the clauses. We refer to the clauses as $c_{1}, \ldots, c_{m}$. For each clause, we append one further row to $A^{\prime}$, each of them identical of the form

$$
\left(\begin{array}{llll}
0 & \ldots & 0 & *
\end{array}\right)^{T} .
$$

We also append one column to $B^{\prime}$ for each clause, each of length $3 n+1$. For each clause, the entry at row $3 n+1$ is set to $*$. If a clause contains a literal of the form $x_{i}$ (in positive form), we set the entry at row $3 i+1$ to $*$. If it contains a literal $\neg x_{i}$, we set the entry at row $3 i+2$ to $*$. In this way, at most 4 entries in the column are fixed to $*$, and we fix all other entries to be 0 . Continuing the above example, for the clause $x_{0} \vee \neg x_{1} \vee x_{2}$, we obtain a column of the form

$$
\left(\begin{array}{llllllllll}
* & 0 & 0 & 0 & * & 0 & * & 0 & 0 & *
\end{array}\right)
$$

Let $A$ and $B$ denote the matrices extended from $A^{\prime}$ and $B^{\prime}$ with the above procedure. We next define $C$ as a square matrix of dimension $2 n+1+m$ as follows: The upper left $(2 n+1) \times(2 n+1)$ submatrix is set to $I_{2 n+1}$. The rest of the first row is set to 0 , and the rest of the diagonal is set to 1 . All other entries are set to $*$. This concludes the description of a GCI-instance $A B=C$ out of a $3 \mathrm{CNF}$ formula $\phi$. We exemplify the construction for the formula $\left(x_{0} \vee x_{1} \vee \neg x_{2}\right) \wedge\left(\neg x_{0} \vee x_{1} \vee x_{2}\right)$, where the lines mark the boundary of $A^{\prime}$ and $B^{\prime}$, respectively.

$$
\left(\begin{array}{cccccccccc}
a_{0} & b_{0} & 0 & a_{1} & b_{1} & 0 & a_{2} & b_{2} & 0 & * \\
* & 0 & * & 0 & 0 & 0 & 0 & 0 & 0 & 0 \\
0 & * & * & 0 & 0 & 0 & 0 & 0 & 0 & 0 \\
0 & 0 & 0 & * & 0 & * & 0 & 0 & 0 & 0 \\
0 & 0 & 0 & 0 & * & * & 0 & 0 & 0 & 0 \\
0 & 0 & 0 & 0 & 0 & 0 & * & 0 & * & 0 \\
0 & 0 & 0 & 0 & 0 & 0 & 0 & * & * & 0 \\
\hline 0 & 0 & 0 & 0 & 0 & 0 & 0 & 0 & 0 & * \\
0 & 0 & 0 & 0 & 0 & 0 & 0 & 0 & 0 & *
\end{array}\right)\left(\begin{array}{ccccccc|cc}
0 & * & 0 & 0 & 0 & 0 & 0 & * & 0 \\
0 & 0 & * & 0 & 0 & 0 & 0 & 0 & * \\
0 & * & * & 0 & 0 & 0 & 0 & 0 & 0 \\
0 & 0 & 0 & * & 0 & 0 & 0 & * & * \\
0 & 0 & 0 & 0 & * & 0 & 0 & 0 & 0 \\
0 & 0 & 0 & * & * & 0 & 0 & 0 & 0 \\
0 & 0 & 0 & 0 & 0 & * & 0 & 0 & * \\
0 & 0 & 0 & 0 & 0 & 0 & * & * & 0 \\
0 & 0 & 0 & 0 & 0 & * & * & 0 & 0 \\
* & 0 & 0 & 0 & 0 & 0 & 0 & * & * \\
& & & & & & & &
\end{array}\right.
$$




$$
=\left(\begin{array}{lllllllll}
1 & 0 & 0 & 0 & 0 & 0 & 0 & 0 & 0 \\
0 & 1 & 0 & 0 & 0 & 0 & 0 & * & * \\
0 & 0 & 1 & 0 & 0 & 0 & 0 & * & * \\
0 & 0 & 0 & 1 & 0 & 0 & 0 & * & * \\
0 & 0 & 0 & 0 & 1 & 0 & 0 & * & * \\
0 & 0 & 0 & 0 & 0 & 1 & 0 & * & * \\
0 & 0 & 0 & 0 & 0 & 0 & 1 & * & * \\
* & * & * & * & * & * & * & 1 & * \\
* & * & * & * & * & * & * & * & 1
\end{array}\right)
$$

Lemma $4 A B=C$ admits a solution if and only if $\phi$ is satisfiable.

Proof " $\Rightarrow$ ": Let us assume that $A B=C$ has a solution, which also implies a solution $A^{\prime} B^{\prime}=I_{2 n+1}$ being a subproblem encoded in the instance. Fixing a solution, we assign an assignment of the variables of $\phi$ as follows: If the entry $A_{1,3 i+1}$ is non-zero, we set $x_{i}$ to true. If the entry $A_{1,3 i+2}$ is non-zero, we set $x_{i}$ to false. If neither is non-zero, we set $x_{i}$ to false as well (the choice is irrelevant). Note that by Lemma 3 , not both $A_{1,3 i+1}$ and $A_{1,3 i+2}$ can be non-zero, so the assignment is well-defined.

First of all, let $\gamma$ be the rightmost entry of the first row of $A$. Because the $(1,1)$-entry of $C$ is set to 1 , it follows that $\gamma \delta=1$, where $\delta$ is the lowest entry of the first column of $B$. Hence, in the assumed solution, $\gamma \neq 0$.

Now fix a clause $c$ in $\phi$ and let $v$ denote the column of $B$ assigned to this clause, with column index $i$. Recall that $v$ consists of (up to) three $*$ entries chosen according to the literals of $c$, and a $*$ entry at the lowest position. Let $\lambda$ denote the value of that lowest entry in the assumed solution of $A B=C$. We see that $\lambda \neq 0$, with a similar argument as for $\gamma$ above, using the $(i, i)$-entry of $C$.

Now, the $(1, i)$ entry of $C$ is set to 0 by construction which yields a constraint of the form

$$
\mu_{1} v_{1}+\mu_{2} v_{2}+\mu_{3} v_{3}+\underbrace{\gamma \lambda}_{\neq 0}=0
$$

where $v_{1}, v_{2}, v_{3}$ are entries of $v$ at the $*$ positions, and $\mu_{1}, \mu_{2}, \mu_{3}$ the corresponding entries of the first row of $A$. We observe that at least one term $\mu_{j} v_{j}$ must be non-zero, hence both entries are non-zero.

This implies that the chosen assignment satisfies the clause: if $v_{j}$ is at index $3 k+1$ for some $k$, the clause contains the literal $x_{k}$ by construction and since $\mu_{j} \neq 0$, our assignment sets $x_{k}$ to true. The same argument applies to $v_{j}$ of the form $3 k+2$. It follows that the assignment satisfies all clauses and hence, $\phi$ is satisfiable.

" $\Leftarrow$ ": We pick a satisfying assignment for $\phi$ and fill the first row of $A$ as follows: if $x_{i}$ is true, we set $\left(A_{1,3 i+1}, A_{1,3 i+2}\right)$ to $(1,0)$ if $x_{i}$ is false, we set it as $(0,1)$. By Lemma 3, there exists a solution for $A^{\prime} B^{\prime}=I_{2 n+1}$ with this initial values and we choose such a solution, filling the upper $(2 n+1)$ rows of $A$ and the left $(2 n+1)$ columns of $B$. Note that similar as above, the value $\gamma$ at $A_{1,3 n+1}$ must be non-zero in such a solution. In the remaining $m$ rows of $A$, by construction, we only need to 
pick the rightmost entry, and we set it to $\gamma$ in each of these rows. That determines all entries of $A$.

To complete $B^{\prime}$ to $B$, we need to fix values in the columns of $B$ associated to clauses. In each such column, we pick the lowest entry to be $\frac{1}{\gamma}$, satisfying the constraints of $C$ along the diagonal. Fixing a column $i$ of $B$, the $(1, i)$-constraint of $C$ reads as

$$
\mu_{1} v_{1}+\mu_{2} v_{2}+\mu_{3} v_{3}+\underbrace{\gamma \frac{1}{\gamma}}_{=1}=0,
$$

where $v_{1}, v_{2}, v_{3}$ are the remaining non-zero entries in $i$-th column. Because we encoded a satisfying assignment of $\phi$ in the first row of $A$, at least one $\mu_{j}$ entry is 1 . We set the corresponding entry $v_{j}$ to -1 , and the remaining $v_{k}$ 's to 0 . In this way, all constraints are satisfied, and the GCI-instance has a solution.

Clearly, the GCI-instance of the preceding proof can be computed from $\phi$ in polynomial time. It follows:

Theorem 1 CI is NP-complete.

Proof Lemma 4 shows the reduction of 3SAT to GCI, proving that GCI is NP-complete. As shown in Lemma 2, GCI reduces to CI, proving the claim.

\section{Modules and Interleavings}

In what follows, all vector spaces are understood to be $\mathbb{F}$-vector spaces for the fixed base field $\mathbb{F}$. Also, for points $p=\left(p_{x}, p_{y}\right), q=\left(q_{x}, q_{y}\right)$ in $\mathbb{R}^{2}$, we write $p \leq q$ if $p_{x} \leq q_{x}$ and $p_{y} \leq q_{y}$.

\subsection{Persistence Modules}

A (two-parameter) persistence module $M$ is a collection of $\mathbb{F}$-vector spaces $V_{p}$, indexed over $p \in \mathbb{R}^{2}$ together with linear maps $M_{p \rightarrow q}$ whenever $p \leq q$. These maps must have the property that $M_{p \rightarrow p}$ is the identity map on $M_{p}$ and $M_{q \rightarrow r} \circ M_{p \rightarrow q}=$ $M_{p \rightarrow r}$ for $p \leq q \leq r$. Much more succinctly, a persistence module is a functor from the poset category $\mathbb{R}^{2}$ to the category of vector spaces. A morphism between $M$ and $N$ is a collection of linear maps $\left\{f_{p}: M_{p} \rightarrow N_{p}\right\}$ such that $N_{p \rightarrow q} \circ f_{p}=f_{q} \circ M_{p \rightarrow q}$. We say that $f$ is an isomorphism if $f_{p}$ is an isomorphism for all $p$, and denote this by $M \cong N$. If we view persistence modules as functors, a morphism is simply a natural transformation between the functors.

The simplest example is the 0 -module where $M_{p}$ is the trivial vector space for all $p \in \mathbb{R}^{2}$. For a more interesting example, define an interval in the poset $\left(\mathbb{R}^{2}, \leq\right)$ to be a non-empty subset $S \subset \mathbb{R}^{2}$ such that whenever $a, c \in S$ and $a \leq b \leq c$, then $b \in S$, and moreover, if $a, c \in S$, there exists a sequence of elements $a=b_{1}, \ldots, b_{\ell}=c$ of elements in $S$ such that $b_{i} \leq b_{i+1}$ or $b_{i+1} \leq b_{i}$. We associate an interval module $I^{S}$ 
Fig. 1 A staircase of size 3 (shaded area)

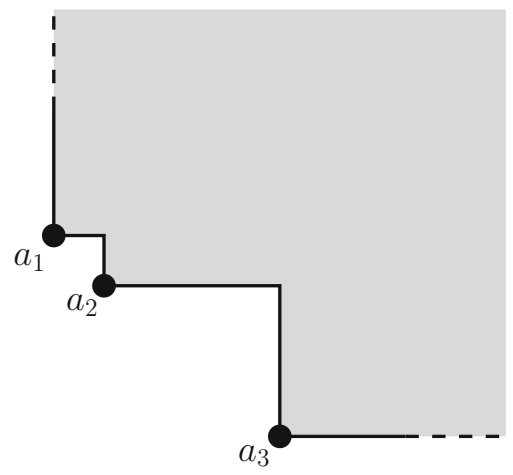

to $S$ as follows: for $p \in S$, we set $I_{p}^{S}:=\mathbb{F}$, and $I_{p}^{S}:=0$ otherwise. As map $I_{p \rightarrow q}^{S}$ with $p \leq q$, we attach the identity map if $p, q \in S$, and the 0 -map otherwise.

For $a \in \mathbb{R}^{2}$, let $\langle a\rangle:=\left\{x \in \mathbb{R}^{2} \mid a \leq x\right\}$ be the infinite rectangle with $a$ as lower-left corner. Given $k$ elements $a_{1}, \ldots, a_{k} \in \mathbb{R}^{2}$, the set

$$
S:=\bigcup_{i=1, \ldots, k}\left\langle a_{i}\right\rangle
$$

is called the staircase with elements $a_{1}, \ldots, a_{k}$. We call $k$ the size of the staircase. See Fig. 1 for an illustration. It is easy to verify that $S$ is an interval for $k \geq 1$. Clearly, if $a_{i} \leq a_{j}$, we can remove $a_{j}$ without changing the staircase, so we assume that the elements forming the staircase are pairwise incomparable. The staircase module is the interval module associated to the staircase.

Given two persistence modules $M$ and $N$, the direct sum $M \oplus N$ is the persistence module where $(M \oplus N)_{p}:=M_{p} \oplus N_{p}$, and the linear maps are defined componentwise in the obvious way. We call a persistence module $M$ indecomposable, if in any decomposition $M=M_{1} \oplus M_{2}, M_{1}$ or $M_{2}$ is the 0 -module. For example, it is not difficult to see that interval modules are indecomposable. We call $M$ interval decomposable if $M$ admits a decomposition $M \cong M_{1} \oplus \ldots \oplus M_{\ell}$ into (finitely many) interval modules. The decomposition of any persistence module into interval modules is unique up to rearrangement and isomorphism of the summands; see [5, Section 2.1] and the references therein. This implies that there is a well-defined multiset of intervals $B(M)$ given by the decomposition of $M$ into interval modules. The multiset $B(M)$ is called the barcode of $M$. Not every module is interval decomposable; we remark that already rather simple geometric constructions can give rise to complicated indecomposable elements [7].

\subsection{Interleavings}

Let $\epsilon \in \mathbb{R}$. For a persistence module $M$, the $\epsilon$-shift of $M$ is the module $M^{\epsilon}$ defined by $M_{p}^{\epsilon}=M_{p+\epsilon}$ (where $p+\epsilon=\left(p_{x}+\epsilon, p_{y}+\epsilon\right)$ ) and $M_{p \rightarrow q}^{\epsilon}=M_{p+\epsilon \rightarrow q+\epsilon}$. Note that $\left(M^{\epsilon}\right)^{\delta}=M^{\epsilon+\delta}$. As an example, staircase modules are closed under shift: the

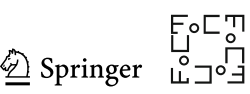


$\epsilon$-shift of the staircase module associated to $\bigcup\left\langle a_{i}\right\rangle$ is the staircase module associated to $\bigcup\left\langle a_{i}-\epsilon\right\rangle$. We can also define shift on morphisms: for $f: M \rightarrow N, f^{\epsilon}: M^{\epsilon} \rightarrow N^{\epsilon}$ is given by $f_{p}^{\epsilon}=f_{p+\epsilon}$. For $\epsilon \geq 0$, there is an obvious morphism $\operatorname{Sh}_{M}(\epsilon): M \rightarrow M^{\epsilon}$ given by the internal morphisms of $M$, that is, we have $\operatorname{Sh}_{M}(\epsilon)_{p}=M_{p \rightarrow p+\epsilon}$. In practice we will often suppress notation and simply write $M \rightarrow M^{\epsilon}$ for this morphism.

With this in mind, we define an $\epsilon$-interleaving between $M$ and $N$ for $\epsilon \geq 0$ as a pair $(f, g)$ of morphisms $f: M \rightarrow N^{\epsilon}$ and $g: N \rightarrow M^{\epsilon}$ such that $g^{\epsilon} \circ f=\operatorname{Sh}_{M}(2 \epsilon)$ and $f^{\epsilon} \circ g=\mathrm{Sh}_{N}(2 \epsilon)$. Concretely, an $\epsilon$-interleaving between two persistence modules $M$ and $N$ is a collection of maps

$$
\begin{aligned}
& f_{p}: M_{p} \rightarrow N_{p+\epsilon} \\
& g_{p}: N_{p} \rightarrow M_{p+\epsilon}
\end{aligned}
$$

such that all diagrams that can be composed out of the maps $f_{*}, g_{*}$, and the linear maps of $M$ and $N$ commute. Note that a 0 -interleaving simply means that the persistence modules are isomorphic. Also, an $\epsilon$-interleaving induces a $\delta$-interleaving for $\epsilon<\delta$ directly by a suitable composition with the linear maps of the modules.

We say that two modules are $\epsilon$-interleaved if there exists an $\epsilon$-interleaving between them. We define the interleaving distance of two modules $M$ and $N$ as

$$
d_{I}(M, N):=\inf \{\epsilon \geq 0 \mid M \text { and } N \text { are } \epsilon \text {-interleaved }\} .
$$

Note that $d_{I}$ defines an extended pseudometric on the space of persistence modules. The distance between two modules might be infinite, and there are non-isomorphic modules with distance 0 . The triangle inequality follows from the simple observation that an $\epsilon$-interleaving between $M_{1}$ and $M_{2}$ and a $\delta$-interleaving between $M_{2}$ and $M_{3}$ can be composed to an $(\epsilon+\delta)$-interleaving between $M_{1}$ and $M_{3}$.

\subsection{Representation of Persistence Modules}

For studying the computational complexity of the interleaving distance, we need to specify a finite representation of persistence modules that allows us to pass such modules as an input to an algorithm.

A graded matrix representation of a module $M$ is a 3-tuple $(G, R, A)$, where $G=\left\{g_{1}, \ldots, g_{n}\right\}$ is a list of $n$ points in $\mathbb{R}^{2}, R=\left\{r_{1}, \ldots, r_{m}\right\}$ is a list of $m$ points in $\mathbb{R}^{2}$, (with repetitions allowed), and $A$ is an $(m \times n)$-matrix over the base field $\mathbb{F}$. Equivalently, we can simply think of a matrix $A$ where each row and column is annotated with a grading in $\mathbb{R}^{2}$.

The algebraic explanation for this representation is as follows: it is known that a persistence module $M$ over $\mathbb{R}^{2}$ can be equivalently described as a graded $\mathcal{R}$-module over a suitably chosen ring $\mathcal{R}$. Assuming that $M$ is finitely presented, we can consider the free resolution of $M$

$$
\mathcal{R}^{m} \stackrel{\partial^{T}}{\rightarrow} \mathcal{R}^{n} \rightarrow M \rightarrow 0
$$


A graded matrix representation is simply a way to encode the map $\partial$ in this resolution.

Let us describe for concreteness how a representation $(G, R, A)$ gives rise to a persistence module. First, let $\mathbb{F}_{1}, \ldots, \mathbb{F}_{n}$ be copies of $\mathbb{F}$, and let $e_{i}$ be the 1-element of $\mathbb{F}_{i}$. For $p \in \mathbb{R}^{2}$, we define $\operatorname{Gen}_{p}$ as the direct sum of all $\mathbb{F}_{i}$ such that $g_{i} \leq p$. Moreover, every row of $A$ gives rise to a linear combination of the entries $e_{1}, \ldots, e_{n}$. Let $c_{i}$ denote the linear combination in row $i$. We define $\operatorname{Rel}_{p}$ to be the span of all linear combinations $c_{i}$ for which $r_{i} \leq p$. Then, we set

$$
M_{p}:=\frac{\operatorname{Gen}_{p}}{\operatorname{Rel}_{p}}
$$

which is a $\mathbb{F}$-vector space. For $p \leq q$, writing $[x]_{p}$ for an element of $M_{p}$ with $x \in \operatorname{Gen}_{p}$, we define

$$
M_{p \rightarrow q}\left([x]_{p}\right):=[x]_{q} .
$$

It is easy to check that that $[x]_{q}$ is well-defined ( since Gen $_{p} \subseteq \operatorname{Gen}_{q}$ ) and independent of the chosen representative in $\operatorname{Gen}_{p}$ ( since $\operatorname{Rel}_{p} \subseteq \operatorname{Rel}_{q}$ ). Moreover, it is straightforward to verify that these maps satisfy the properties of a persistence module.

In short, every persistence module that can be expressed by finitely many generators and relations can be brought into graded matrix representation. For instance, a staircase module for $a_{1}, \ldots, a_{n}$ of size $n$ where the $a_{i}$ are ordered by increasing first coordinate can be represented by a matrix with $n$ columns graded by $a_{1}, \ldots, a_{n}$, and $n-1$ rows, where every row corresponds to a pair $(i, i+1)$ with $1 \leq i \leq n-1$. In this row, we encode the relation $e_{i}=e_{i+1}$ and grade it by $p_{i j}$, which is the (unique) minimal element $q$ in $\mathbb{R}^{2}$ such that $a_{i} \leq q$ and $a_{i+1} \leq q$. Hence, the graded matrix representation of a staircase of size $n$ has a size that is polynomial in $n$.

We also remark that a graded matrix representation is equivalent to free implicit representations [15, Sec 5.1] for the special case of $m_{0}=0$.

\section{Hardness of Interleaving Distance}

We consider the following computational problems:

1-INTERLEAVING: Given two persistence modules $M, N$ in graded matrix representation, decide whether they are 1-interleaved.

$c$ - APPROX- INTERLEAVING- DiSTANCE: Given two persistence modules $M, N$ in graded matrix representation, return a real number $r$ such that

$$
d_{I}(M, N) \leq r \leq c \cdot d_{I}(M, N)
$$

Obviously, the problem of computing $d_{I}(M, N)$ exactly is equivalent to the above definition with $c=1$.

The main result of this section is the following theorem:

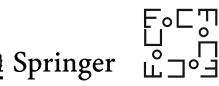


Theorem 2 Given a CI-instance $(n, P, Q)$, we can compute in polynomial time in $n$ a pair of persistence modules $(M, N)$ in graded matrix representation such that

$$
d_{I}(M, N)=\left\{\begin{array}{ll}
1 & \text { if }(n, P, Q) \in C I \\
3 & \text { if }(n, P, Q) \notin C I
\end{array} .\right.
$$

Moreover, both $M$ and $N$ are direct sums of staircase modules and hence interval decomposable.

We will postpone the proof of Theorem 2 to the end of the section and first discuss its consequences.

Theorem 3 1-INTERLEAVING is NP-complete.

Proof We first argue that 1-INTERLEAVING is in NP. First, note that to specify a 1interleaving, it suffices to specify the maps at the points in $S$, where $S$ is a finite set whose size is polynomial in the size of the graded matrix representation. More precisely, $S$ contains the critical grades of the two modules (that is, the grades specified by $G$ and $R$ ), as well as the least common successors of such elements. That ensures that every vector space (in both modules) can be isomorphically pulled back to one of the elements of $S$, and the interleaving map can be defined using this pull-back. It is enough to consider the points in $S$ to check whether this set of pointwise maps is a valid morphism.

We can furthermore argue that verifying that a pair of such maps yields a 1interleaving can be checked in a polynomial number of steps. Again, this involves mostly the maps specified above, as well as the corresponding maps shifted by $(1,1)$, in order to check the compatibility of the two interleaving maps. We omit further details of this step.

Finally, 1-INTERLEAVING is NP-hard: Assuming a polynomial time algorithm $A$ to decide the problem, we can design a polynomial time algorithm for CI just by transforming $(n, P, Q)$ into a pair of modules $(M, N)$ using the algorithm from Theorem 2 . If $A$ applied on $(M, N)$ returns true, we return that $(n, P, Q)$ is in CI. Otherwise, we return that $(n, P, Q)$ is not in CI. Correctness follows from Theorem 2, and the algorithm runs in polynomial time, establishing a polynomial time reduction. By Theorem 1, CI is NP-hard, hence, so is 1-INTERLEAVING.

Theorem $4 c$-APPROX- INTERLEAVING- DISTANCE is NP-hard for every $c<3$ (i.e., $a$ polynomial time algorithm for the problem implies $P=N P$ ).

Proof Fixing $c<3$, assuming a polynomial time algorithm $A$ for $c$-APPROXINTERLEAVING- DISTANCE yields a polynomial time algorithm for CI: Given the input $(n, P, Q)$, we transform it into $(M, N)$ with Theorem 2 . Then, we apply $A$ on $(M, N)$. If the result is less than 3 , we return that $(n, P, Q)$ is in CI. Otherwise, we return that $(n, P, Q)$ is not in CI. Correctness follows from Theorem 2, noting that if $(n, P, Q)$ is in CI, algorithm $A$ must return a number in the interval [1,c] and $c<3$ is assumed. If $(n, P, Q)$ is not in $\mathrm{CI}$, it returns a number $\geq 3$. Also, the algorithm runs in polynomial time in $n$. Therefore, the existence of $A$ yields a polynomial time algorithm for CI, implying $\mathrm{P}=\mathrm{NP}$ with Theorem 1 . 
Since the modules in Theorem 2 are direct sums of staircases, both Theorem 3 and Theorem 4 hold already for the restricted case that the modules are interval decomposable.

\subsection{Interleavings of Staircases}

The persistence modules constructed for the proof of Theorem 2 will be direct sums of staircases. Before defining them, we establish some properties of the interleaving map between staircases and their direct sums which reveal the connection to the CI problems.

Recall from Sect. 3 that a morphism $M \rightarrow N$ can be described more concretely as a collection of maps $M_{p} \rightarrow N_{p}$ that are compatible with the linear maps in $M$ and $N$, that $M^{\epsilon}$ is defined by $M_{p}^{\epsilon}=M_{p+\epsilon}$, and that an $\epsilon$-interleaving is a pair of morphisms $\phi: M \rightarrow N^{\epsilon}, \psi: N \rightarrow M^{\epsilon}$ satisfying certain conditions. For staircase modules, the set of morphisms is quite limited.

For $M$ and $N$ staircase modules and $\lambda \in \mathbb{F}$, we denote by $1 \mapsto \lambda$ the collection of linear maps $\phi_{p}$ such that $\phi_{p}(1)=\lambda$ for all $p$ such that $M_{p}=\mathbb{F}$.

Lemma 5 Let $M$ and $N$ be staircase modules. Every morphism from $M$ to $N$ is of the form $1 \mapsto \lambda$ for some $\lambda \in \mathbb{F}$.

Proof Assume first that $p \leq q$ and $M_{p}=\mathbb{F}$. Write $\lambda:=\phi_{p}(1)$. Then, also $M_{q}=\mathbb{F}$, and $\phi_{q}(1)=\lambda$ as well, since the linear maps from $p$ to $q$ for $M$ and $N$ are injective maps.

For incomparable $p$ and $q \in \mathbb{R}^{2}$, we consider the least common successor $r$ of $p$ and $q$. Using the above property twice, we see at once that $\phi_{p}(1)=\phi_{r}(1)=\phi_{q}(1)$.

We examine next which values of $\lambda$ are possible for a concrete pair of staircases. For a staircase $S$, let $S^{\epsilon}$ denote the staircase where each point is shifted by $(\epsilon, \epsilon)$. This way, if $M$ is the module associated to $S, M^{\epsilon}$ is the module associated to $S^{\epsilon}$. As we noted before, the shift of a staircase module is also a staircase module. Define the directed shift distance from the staircase $S$ to the staircase $T$ as

$$
d_{S}(S, T):=\min \left\{\epsilon \geq 0 \mid S \subseteq T^{\epsilon}\right\} .
$$

One can show that the set on the right-hand side has a minimum value by using the fact that a staircase is generated by a finite set of elements, so $d_{s}$ is in fact well-defined. Clearly, $d_{s}(S, T) \neq d_{S}(T, S)$ in general. The following simple observation is crucial for our arguments. Let $M, N$ denote the staircase modules induced by $S$ and $T$.

Lemma 6 If $\epsilon<d_{s}(S, T)$, the only morphism from $M$ to $N^{\epsilon}$ is $1 \mapsto 0$. If $\epsilon \geq d_{s}(S, T)$, every choice of $\lambda \in \mathbb{F}$ yields a morphism $1 \mapsto \lambda$ from $M$ to $N^{\epsilon}$.

Proof In the first case, by construction, there exists some $p$ such that $M_{p}=\mathbb{F}$, but $N_{p+\epsilon}=0$. Hence, 0 is the only choice for $\lambda$.

In the second case, $M_{p}=\mathbb{F}$ implies $N_{p+\epsilon}=\mathbb{F}$ as well. It is easy to check that any choice of $\lambda$ yields a compatible collection of maps, hence a morphism.

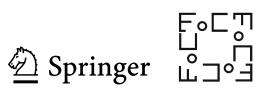


In particular, there are morphisms $M \rightarrow N$ given by arbitrary elements of $\mathbb{F}$ if and only if $S \subseteq T$. As a consequence, we can characterize morphisms of direct sums of staircase modules.

Lemma 7 Let $M=\oplus_{i=1}^{n} M_{i}$ and $N=\oplus_{j=1}^{n} N_{j}$ be direct sums of staircase modules. Then a collection of maps $\phi_{p}: M_{p} \rightarrow N_{p}$ is a morphism if and only if the restriction to $M_{i}$ and $N_{j}$ is a morphism for any $i, j \in\{1, \ldots, n\}$. Therefore, a morphism $\phi$ is determined by an $(n \times n)$-matrix with entries in $\mathbb{F}$.

Proof Let $p \leq q$, and consider the following diagram:

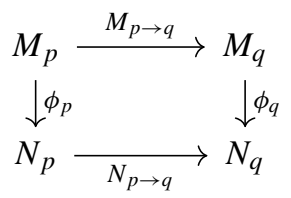

We have $M_{p}=\oplus_{i=1}^{n}\left(M_{i}\right)_{p}$ and $N_{q}=\oplus_{j=1}^{n}\left(N_{j}\right)_{q}$. Thus the diagram above commutes if and only if for all $i$ and $j$, the restrictions of the two compositions to $\left(M_{i}\right)_{p}$ and $\left(N_{j}\right)_{q}$ are the same, since a linear transformation is determined by what happens on basis elements. This is again equivalent to the following diagram commuting for all $i$ and $j$, where $\left(\phi_{i}^{j}\right)_{p}$ is the restriction of $\phi_{p}$ to $M_{i}$ and $N_{j}$ :

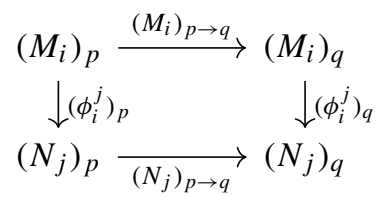

But the collection of $\phi_{p}$ forms a morphism if and only if the first diagram commutes for all $p \leq q$, and the restriction of $\phi_{p}$ to $M_{i}$ and $N_{j}$ forms a morphism if and only if the second diagram commutes for all $p \leq q$. Thus we have proved the desired equivalence.

Observe that the matrix described in Lemma 6 is simply $\phi_{p}: \oplus_{i=1}^{n}\left(M_{i}\right)_{p} \rightarrow$ $\oplus_{j=1}^{n}\left(N_{j}\right)_{p}$ written as a matrix in the natural way for any $p$ contained in the support of $M_{i}$ for all $i$.

Lemma 8 Let $M, N$ be direct sums of staircase modules as above and $\phi: M \rightarrow N^{\epsilon}$ and $\psi: N \rightarrow M^{\epsilon}$ be morphisms. Then $\phi$ and $\psi$ form an $\epsilon$-interleaving if and only if their associated $(n \times n)$-matrices are inverse to each other.

Proof The composition $\psi^{\epsilon} \circ \phi: M \rightarrow M^{2 \epsilon}$ is represented by the matrix $B A$, as one can see by restricting to a single point contained in all relevant staircases as in the observation above. The morphism $\operatorname{Sh}_{M}(2 \epsilon): M \rightarrow M^{2 \epsilon}$ is represented by the identity matrix. By definition, $(\phi, \psi)$ is an interleaving if and only if these are equal and the corresponding statement holds for $\phi^{\epsilon} \circ \psi$, so the statement follows.

As a consequence, we obtain the following intermediate result. 
Theorem 5 Let $(n, P, Q)$ be a $C I$-instance and let $S_{1}, \ldots, S_{n}, T_{1}, \ldots, T_{n}$ be staircases such that

$$
d_{S}\left(S_{i}, T_{j}\right)=\left\{\begin{array}{ll}
3 & \text { if }(i, j) \in P \\
1 & \text { if }(i, j) \notin P
\end{array} \quad d_{S}\left(T_{j}, S_{i}\right)= \begin{cases}3 & \text { if }(j, i) \in Q \\
1 & \text { if }(j, i) \notin Q\end{cases}\right.
$$

Write $M_{i}, N_{j}$ for the modules associated to $S_{i}, T_{j}$, respectively, and $M:=\oplus M_{i}$ and $N:=\oplus N_{j}$. Then

$$
d_{I}(M, N)= \begin{cases}1 & \text { if }(n, P, Q) \in C I \\ 3 & \text { if }(n, P, Q) \notin C I\end{cases}
$$

Proof Assume first that $(n, P, Q) \in C I$. Let $A, B$ be a solution. We show that $A$ and $B$ define morphisms from $M$ to $N^{1}$ and from $N$ to $M^{1}$. We restrict to the map from $M$ to $N^{1}$, as the other case is symmetric. By Lemma 7, it suffices to show that the map from $M_{i}$ to $N_{j}^{1}$ is a morphism. This map is represented by the entry $A_{i j}$. If $(i, j) \in P, A_{i j}=0$ by assumption, and the 0-map is always a morphism. If $(i, j) \notin P, d_{S}\left(S_{i}, T_{j}\right)=1$ by construction. Hence, by Lemma 6 any field element yields a morphism. This shows that $A$ and $B$ define a pair of valid morphisms, and by Lemma 8 this pair is an 1-interleaving, as $A B=I_{n}$. Also with Lemma 6 , it can easily be proved that the only morphism $M \rightarrow N^{\epsilon}$ with $\epsilon<1$ is the 0 -map. Hence, $d_{I}(M, N)=1$ in this case.

Now assume that $(n, P, Q) \notin C I$. It is clear that $M$ and $N$ as constructed are 3-interleaved: the matrix $I_{n}$ yields a valid morphism from $M$ to $N^{3}$ and from $N$ to $M^{3}$ with Lemma 6 . Assume for a contradiction that there exists an $\epsilon$-interleaving between $M$ and $N$ represented by matrices $A, B$, with $\epsilon<3$. For $(i, j) \in P$, since $d_{s}\left(M_{i}, N_{j}\right)=3>\epsilon$, Lemma 6 implies that the entry $A_{i, j}$ must be equal to 0. Likewise, $B_{j, i}=0$ whenever $(j, i) \in Q$. By Lemma $8, A B=I_{n}$, and it follows that $A$ and $B$ constitute a solution to the CI-instance $(n, P, Q)$, a contradiction.

\subsection{Construction of the Staircases}

To prove Theorem 2, it suffices to construct staircases $S_{1}, \ldots, S_{n}, T_{1}, \ldots, T_{n}$ with the properties from Theorem 5, in polynomial time.

To describe our construction, we consider to two "base staircases" which we depict in Fig. 2. In what follows, a shift of a point $a$ by $(1,1)$ means replacing $a$ with the point $a-(1,1)$. The base staircase $S$ is formed by the points $(-t, t)$ for $t=$ $-4 n^{2},-4 n^{2}+2, \ldots, 4 n^{2}$, but with the right side (i.e., the points with negative $t$ ) shifted by $(1,1)$. Likewise, the base staircase $T$ consists of the same points, but with the left side shifted by $(1,1)$. We observe immediately that the staircase distance of the two base staircases is equal to 1 in either direction. We call the points defining the staircases corners from now on.

Now we associate to every entry in $P$ a corner in the left side of $S$ (that is, some $(-t, t)$ with $t>0)$. We also associate with the entry a corner in $T$, namely the shifted point $(-t-1, t-1)$. We do this in a way that between two associated corners of

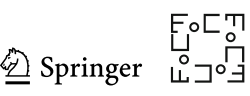




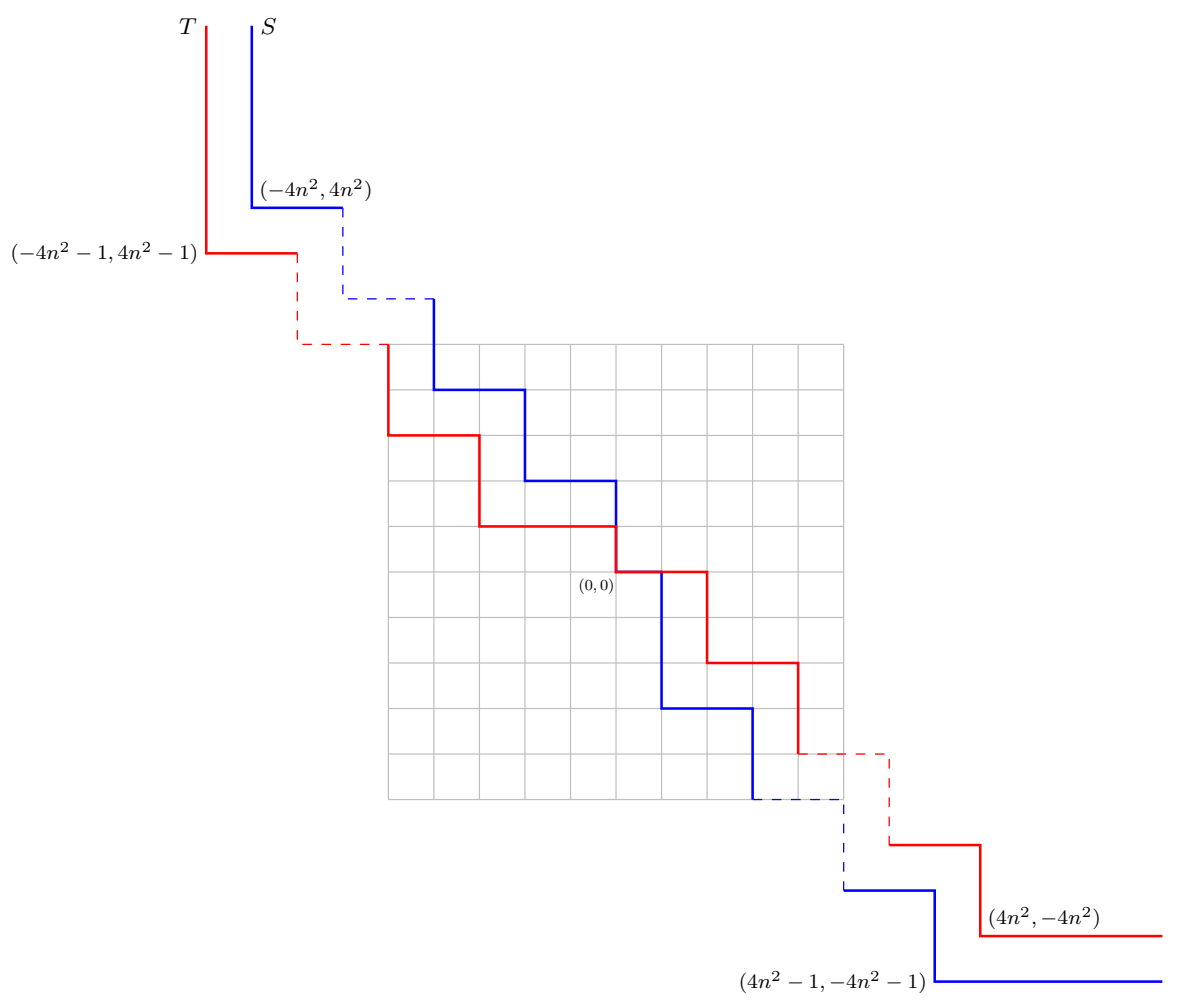

Fig. 2 The base staircases $S$ and $T$

$S$, there is at least one corner of the staircase that is not associated. Note that this is always possible because $|P| \leq n^{2}$ and we have $2 n^{2}$ corners on the left side. We associate corners to entries of $Q$ in the symmetric way, using the right side of the base staircases.

We construct the staircases $S_{i}$ and $T_{j}$ out of the base staircases $S$ and $T$, only shifting associated corners by $(2,2)$ or $(-2,-2)$ according to $P$ and $Q$. Specifically, for the staircase $S_{i}$, we start with $S$ and for any entry $(i, j)$ in $P$, we shift the associated corner of $S$ by $(2,2)$. For every entry $(j, i)$ in $Q$, we shift the associated corner by $(-2,-2)$. The resulting (partially) shifted version of $S$ defines $S_{i}$.

$T_{j}$ is defined symmetrically: for every $(i, j) \in P$, we shift the associated corner by $(-2,-2)$. For every $(j, i) \in Q$, we shift the associated corner by $(2,2)$.

We next analyze the staircase distance of $S_{i}$ and $T_{j}$. We observe that, because there is an unassociated corner in-between any two associated corners, the $\pm(2,2)$ shifts of distinct corners do not interfere with each other. Hence, it suffices to consider the distance of one associated corner of $S_{i}$ to $T_{j}$. Fix the corner $c_{S}$ of $S$ associated to some entry $(k, \ell) \in P$. Let $c_{T}$ denote the associated corner of $T$, that is, $c_{T}=c_{S}-(1,1)$. See Fig. 3 (left) for an illustration. If $k \neq i$ and $\ell \neq j$, neither $c_{S}$ nor $c_{T}$ gets shifted, and since $c_{T} \leq c_{S}$, the shift required from $c_{T}$ to reach $c_{S}$ is 0 . If $k=i$ and $\ell \neq j$, then $c_{S}$ gets shifted by $(2,2)$, and the required shift is 1 (see second picture of Fig. 3 ). 

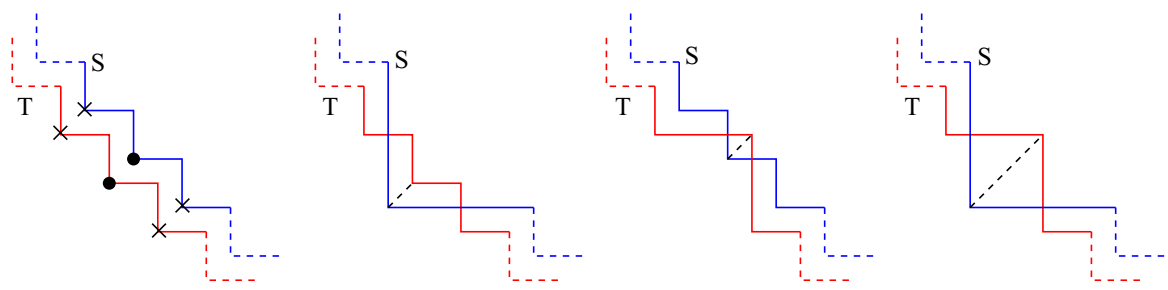

Fig. 3 Left: the associated corners $c_{S}$ (on staircase $S$ ) and $c_{T}$ (on staircase $T$ ) are marked by black circles. The two neighboring corners on both staircases (marked with $\mathrm{x}$ ) are not associated and hence not shifted in the construction. Second and third picture: the cases $(i, \ell)$ with $\ell \neq j$ and $(k, j)$ with $k \neq i$. In both cases, the directed staircase distance is 1 , as illustrated by the dashed line. Right: the case $(i, j)$. In that case, a shift of 3 is necessary to move the corner of $T$ to $S$

If $k \neq i$ and $\ell=j, c_{T}$ gets shifted by $(-2,-2)$, the required shift is also 1 (see 3 rd picture of Fig. 3). If $k=i$ and $\ell=j$, both $c_{S}$ and $c_{T}$ get shifted, and the distance of the shifted $c_{T}$ to reach $c_{S}$ increases to 3 (see 4 th picture of Fig. 3). This argument implies that the (directed) staircase distance from $S_{i}$ to $T_{j}$ is 3 if $(i, j) \in P$, and 1 otherwise. A completely symmetric argument works for $d_{S}\left(T_{j}, S_{i}\right)$, inspecting the corners associated to $Q$.

Finally, it is clear that the size and construction time of each $S_{i}$ and each $T_{j}$ is polynomial in $n$. As remarked at the end of Sect. 3, the staircase module can be brought in graded matrix representation in polynomial time in $n$, and the same holds for the direct sum of these modules. This finishes the proof of Theorem 2.

With the construction of $M$ and $N$ from Theorem 2 fresh in mind, we can explain the obstacles to obtaining a constant bigger than 3. Exchanging 3 with another constant in Theorem 5 is not a problem; the proof would be exactly the same. The trouble is to construct $S_{i}$ and $T_{j}$ satisfying the conditions in Theorem 5 if 3 is replaced by some $\epsilon>3$. In that case, one would have to force $d_{S}\left(S_{i}, T_{j}\right) \geq \epsilon$ for $(i, j) \in P$ and $d_{S}\left(T_{j}, S_{i}\right) \geq \epsilon$ for $(j, i) \in Q$, while still keeping $d_{S}\left(S_{i}, T_{j}\right) \leq 1$ for $(i, j) \notin P$ and $d_{S}\left(T_{j}, S_{i}\right) \leq 1$ for $(j, i) \notin Q$. As we have shown, letting $d_{S}\left(S_{i}, T_{j}\right)=3$ when $(i, j) \in P$ can be done. However, even if $(i, j) \in P$, there might be $i^{\prime}, j^{\prime}$ such that $\left(i, j^{\prime}\right),\left(i^{\prime}, j\right) \notin P$ and $\left(j^{\prime}, i^{\prime}\right) \notin Q$, implying

$$
\begin{aligned}
d_{S}\left(S_{i}, T_{j^{\prime}}\right) & \leq 1, \\
d_{S}\left(T_{j^{\prime}}, S_{i^{\prime}}\right) & \leq 1, \\
d_{S}\left(S_{i^{\prime}}, T_{j}\right) & \leq 1 .
\end{aligned}
$$

which gives $d_{S}\left(S_{i}, T_{j}\right) \leq 3<\epsilon$ by the triangle inequality. This proves that one cannot simply increase the constant in Theorem 5, change the construction of $S_{i}$ and $T_{j}$, and get a better result. That is not to say that using CI problems to improve Theorem 4 is necessarily hopeless, but it would not come as a surprise if a radically new approach is needed, if the theorem can be improved at all.

This is related to questions of stability, more precisely of whether $d_{B}(B(M), B(N))$ $\leq 3 d_{I}(M, N)$ is true for staircase decomposable modules, where $d_{B}$ is the bottleneck distance. We have associated pairs of modules to CI problems in a way such 
that interleavings correspond to solutions of the CI problems. Matchings between the barcodes of the modules (which is what gives rise to the bottleneck distance) correspond to solutions to the CI problems of a particular simple form, namely with a single non-zero entry in each column and row of each matrix. Claiming that $d_{B}(B(M), B(N)) \leq 3 d_{I}(M, N)$ is then related to claiming that if a CI problem has a solution, then a "weakening" of the CI problem has a solution of this simple form. We will not go into details about this, other than to say that there are questions that can be formulated purely in terms of CI problems whose answers could have very interesting consequences for the study of interleavings, also beyond the work done in this paper.

\section{Indecomposable Modules}

Fix a CI problem $(n, P, Q)$ as in the previous section and let $M$ and $N$ be the associated persistence modules. We shall now construct two indecomposable persistence modules $\widehat{M}$ and $\widehat{N}$ such that $\widehat{M}$ and $\widehat{N}$ are $\epsilon$-interleaved if and only if $M$ and $N$ are $\epsilon$-interleaved. In what follows we construct $\widehat{M}$; the construction of $\widehat{N}$ is completely analogous.

Recall that a staircase module can be described by a set of generators, or corners. Let $u=(x, x)$ be a point larger than all the corners defining the staircases making up $M$ and $N$. Observe the following: $\operatorname{dim} M_{u}=n, M_{u \rightarrow p}$ is the identity morphism for any $p \geq u$.

Let $s_{i}=x+7+i /(n+1),{ }^{1}$ for $0 \leq i \leq n+1$. Define $\widehat{M}$ at $p \in \mathbb{R}^{2}$ as follows

$$
\widehat{M}_{p}= \begin{cases}0 & \text { if } p \geq\left(s_{i}, s_{n+1-i}\right) \text { for some } 0 \leq i \leq n+1 \\ \mathbb{F} & \text { if } p \in\left[s_{i}, s_{i+1}\right) \times\left[s_{n-i}, s_{n-i+1}\right) \text { for some } 0 \leq i \leq n \\ M_{p} & \text { otherwise. }\end{cases}
$$

Trivially, $\widehat{M}_{p \rightarrow q}$ is the 0 morphism if $\widehat{M}_{p}=0$ or $\widehat{M}_{q}=0$. For $p \leq q$ such that $M_{p}=$ $\widehat{M}_{p}$ and $M_{q}=\widehat{M}_{q}$, let $\widehat{M}_{p \rightarrow q}=M_{p \rightarrow q}$, and for $p, q \in\left[s_{i}, s_{i+1}\right) \times\left[s_{n-i}, s_{n-i+1}\right)$ let $\widehat{M}_{p \rightarrow q}=1_{\mathbb{F}}$. It remains to consider the case that $M_{p}=\widehat{M}_{p}$ and $q \in\left[s_{i}, s_{i+1}\right) \times$ $\left[s_{n-i}, s_{n-i+1}\right)$ for some $i$. Observe that all the internal morphisms are fully specified once we define $\widehat{M}_{u \rightarrow q}$. Indeed, if $p \geq u$, then $\widehat{M}_{u \rightarrow p}$ is the identity, which forces $\widehat{M}_{p \rightarrow q}=\widehat{M}_{u \rightarrow q}$. For any other $p$ we can always choose an $r \geq p$ such that $r \geq u$ and $M_{r}=\widehat{M}_{r}$. The morphism $\widehat{M}_{p \rightarrow q}$ is then given by $\widehat{M}_{p \rightarrow q}=\widehat{M}_{r \rightarrow q} \circ \widehat{M}_{p \rightarrow r}=$ $\widehat{M}_{u \rightarrow q} \circ \widehat{M}_{p \rightarrow r}$. We conclude by specifying the following morphism

$$
\widehat{M}_{u \rightarrow q}= \begin{cases}\left.\pi_{i} \text { (projection onto coordinate } i\right) & \text { if } 1 \leq i \leq n \\ \pi_{1}+\pi_{2}+\ldots+\pi_{n} & \text { if } i=0\end{cases}
$$

Observe that we have a morphism $\pi^{M}: M \rightarrow \widehat{M}$ given by

$$
\pi_{p}^{M}= \begin{cases}\text { id } & \text { if } M_{p}=\widehat{M}_{p} \\ \widehat{M}_{u \rightarrow p} & \text { otherwise }\end{cases}
$$

$\overline{17 \text { can be replaced with any } \delta>6}$. 
Lemma 9 The persistence module $\widehat{M}$ is indecomposable.

Proof We recall the following useful trick: if $M$ is not indecomposable, say $M \cong$ $M^{\prime} \oplus M^{\prime \prime}$, then the projections $M \rightarrow M^{\prime}$ and $M \rightarrow M^{\prime \prime}$ define morphisms which are not given by multiplication with a scalar. Hence, it suffices to show that any endomorphism $\phi: \widehat{M} \rightarrow \widehat{M}$ is multiplication by a scalar. Furthermore, observe that any endomorphism $\phi$ of $\widehat{M}$ is completely determined by $\phi_{u}$. Let $e_{i} \in \mathbb{F}^{n}$ denote the vector $(0, \ldots, 0,1,0, \ldots, 0)$ where the non-zero entry appears at the $i$-th index. For $0 \leq i \leq n, \phi$ must be such that the following diagram commutes

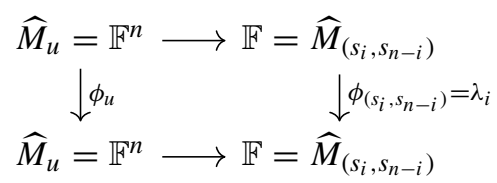

For $1 \leq i \leq n$ this yields that

$$
\pi_{i}\left(\phi_{u}\left(e_{j}\right)\right)= \begin{cases}\lambda_{i} \in \mathbb{F} & \text { if } i=j \\ 0 & \text { if } i \neq j\end{cases}
$$

In particular, we see that $\phi_{u}\left(e_{i}\right)=\lambda_{i} e_{i}$. For $i=0$ we get

$$
\lambda_{0} e_{i}=\lambda_{0} \cdot\left(\pi_{1}+\ldots+\pi_{n}\right)\left(e_{i}\right)=\left(\pi_{1}+\ldots+\pi_{n}\right)\left(\lambda_{i} e_{i}\right)=\lambda_{i} e_{i} .
$$

We conclude that $\lambda_{i}=\lambda_{0}$ and that $\phi_{u}=\lambda_{0} \cdot$ id.

Lemma 10 Fix $1 \leq \epsilon \leq 3 . \widehat{M}$ and $\widehat{N}$ are $\epsilon$-interleaved if and only if $M$ and $N$ are $\epsilon$-interleaved.

Proof Assume that $\phi: M \rightarrow N^{\epsilon}$ and $\psi: N \rightarrow M^{\epsilon}$ form an $\epsilon$-interleaving pair. Define $\widehat{\phi}: \widehat{M} \rightarrow \widehat{N}^{\epsilon}$ and $\widehat{\psi}: \widehat{N} \rightarrow \widehat{M}^{\epsilon}$ by

$$
\widehat{\phi}_{p}=\left\{\begin{array}{ll}
\pi_{p+\epsilon}^{N} \circ \phi_{p} & \text { if } M_{p}=\widehat{M}_{p} \\
0 & \text { otherwise. }
\end{array}, \quad \widehat{\psi}_{p}= \begin{cases}\pi_{p+\epsilon}^{M} \circ \psi_{p} & \text { if } N_{p}=\widehat{N}_{p} \\
0 & \text { otherwise. }\end{cases}\right.
$$

We will show that these two morphisms constitute an $\epsilon$-interleaving pair. Let $p \in \mathbb{R}^{2}$ and consider the following two cases:

1. Assume that $N_{p+\epsilon}=\widehat{N}_{p+\epsilon}$. Under this assumption, we have that $\pi_{p+\epsilon}^{N}=\mathrm{id}$, and thus $\phi_{p}=\widehat{\phi}_{p}$. Using that $\psi$ and $\phi$ form an $\epsilon$-interleaving pair, and that $\pi_{p}^{M}=\mathrm{id}$, we get:

$$
\widehat{\psi}_{p+\epsilon} \circ \widehat{\phi}_{p}=\pi_{p+2 \epsilon}^{M} \circ \psi_{p+\epsilon} \circ \phi_{p}=\pi_{p+2 \epsilon}^{M} \circ M_{p \rightarrow p+2 \epsilon}=\widehat{M}_{p \rightarrow p+2 \epsilon} \circ \pi_{p}^{M}=\widehat{M}_{p \rightarrow p+2 \epsilon} .
$$



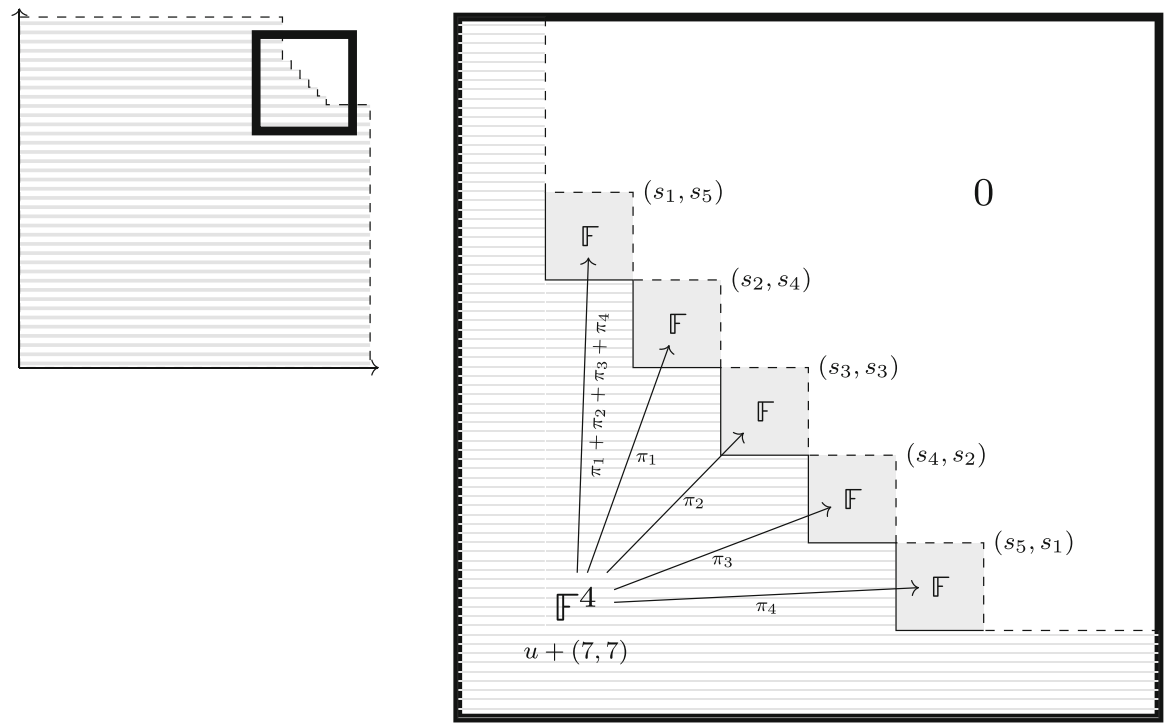

Fig. 4 Left: $\widehat{M}$ coincides with $M$ on the restriction to the shaded subset of $\mathbb{R}^{2}$. Right: this shows the modification done to $M$ in order to obtain an indecomposable persistence module $\widehat{M}$ in the case $n=4$

2. Assume that $N_{p+\epsilon} \neq \widehat{N}_{p+\epsilon}$. Since $\epsilon \geq 1$, it follows by construction that $\widehat{M}_{p+2 \epsilon}=$ 0 . Hence, the interleaving condition is trivially satisfied.

Symmetrically we get that $\widehat{\phi}_{p+\epsilon} \circ \widehat{\phi}_{p}=\widehat{N}_{p \rightarrow p+2 \epsilon}$. Hence, $\widehat{M}$ and $\widehat{N}$ are $\epsilon$-interleaved.

Conversely, assume that $\widehat{\phi}$ and $\widehat{\psi}$ define an interleaving pair between $\widehat{M}$ and $\widehat{N}$. Define $\phi: M \rightarrow N^{\epsilon}$ and $\psi: N \rightarrow M^{\epsilon}$ by

$$
\phi_{p}=\left\{\begin{array}{ll}
\widehat{\phi}_{u} & \text { if } p \geq u \\
\widehat{\phi}_{p} & \text { otherwise }
\end{array}, \quad \psi_{p}= \begin{cases}\widehat{\psi}_{u} & \text { if } p \geq u \\
\widehat{\psi}_{p} & \text { otherwise }\end{cases}\right.
$$

By construction, $\widehat{M}_{p}=M_{p}$ and $\widehat{N}_{p}=N_{p}$ for all $p<u+(7,7)$. This implies that $\widehat{\phi}_{p}=\widehat{\phi}_{u}$ and $\widehat{\psi}_{p}=\widehat{\psi}_{u}$ for all $p<u+(7-\epsilon, 7-\epsilon)$. Hence, for any $p \leq u$ we must have that

$$
\psi_{p+\epsilon} \circ \phi_{p}=\widehat{\psi}_{p+\epsilon} \circ \widehat{\phi}_{p}=\widehat{M}_{p \rightarrow p+2 \epsilon}=M_{p \rightarrow p+2 \epsilon} .
$$

Similarly we get that $\phi_{p+\epsilon} \circ \psi_{p}=N_{p \rightarrow p+2 \epsilon}$ for all such $p$. In particular, by considering the case $p=u$, we see that $\phi_{u}$ and $\psi_{u}$ are mutually inverse matrices. It follows readily that the interleaving condition is satisfied for all $p \not \leq u$.

With the two previous results at hand, we can state the following corollary of Theorem 4.

Corollary 1 1- INTERLEAVING is NP-complete and c-APPROX- INTERLEAVING- DISTANCE is NP-hard for $c<3$, even if the input modules are restricted to indecomposable modules. 
Proof We only prove hardness of 1- INTERLEAVING, the remaining statements follow with the same methods. Given a CI-instance $(n, P, Q)$, we use the construction from Sect. 4 to construct two persistence modules $M$ and $N$. Then we transform them into the indecomposable modules $\widehat{M}$ and $\widehat{N}$ as above. Note that this transformation can be performed in polynomial time in $n$ by introducing up to $n$ relations at the lower-left corners of the $(n+1)$ rectangles in Fig. 4 . Hence, an algorithm to decide 1INTERLEAVING for the case of indecomposable modules would solve CI in polynomial time.

\section{One-Sided Stability}

The results of the previous sections also apply in the setting of one-sided stability. Here we give a brief introduction to the topic; see [1] for a thorough introduction.

Let $f: M \rightarrow N$ be a morphism. The linear map $M_{p \rightarrow q}$ induces a linear map $\operatorname{ker}\left(f_{p}\right) \rightarrow \operatorname{ker}\left(f_{q}\right)$ by restriction, and $N_{p \rightarrow q}$ induces a linear map coker $\left(f_{p}\right) \rightarrow$ $\operatorname{coker}\left(f_{q}\right)$ by taking a quotient, as one can readily verify. We say that $f$ has $\epsilon$-trivial kernel if the map $\operatorname{ker}\left(f_{p}\right) \rightarrow \operatorname{ker}\left(f_{p+\epsilon}\right)$ is the 0 -map for all $p \in \mathbb{R}^{2}$. Likewise, we say that $f$ has $\epsilon$-trivial cokernel if $\operatorname{coker}\left(f_{p}\right) \rightarrow \operatorname{coker}\left(f_{p+\epsilon}\right)$ is the 0 -map for all $p \in \mathbb{R}^{2}$. If $f$ has 0 -trivial kernel (cokernel), then we say that $f$ is injective (surjective). The following lemma follows readily from the definition of an $\epsilon$-interleaving.

Lemma 11 If $f: M \rightarrow N^{\epsilon}$ is an $\epsilon$-interleaving morphism (i.e., it forms an $\epsilon$ interleaving with some $\left.g: N \rightarrow M^{\epsilon}\right)$, then $f$ has $2 \epsilon$-trivial kernel and cokernel.

In fact, Bauer and Lesnick [1] show that in the case of persistence modules over $\mathbb{R}, M$ and $N$ are $\epsilon$-interleaved if and only if there exists a morphism $f: M \rightarrow N^{\epsilon}$ with $2 \epsilon$-trivial kernel and cokernel. They also observe that this equivalence does not generalize to two parameters. However, it is true (and the proof is very similar to the one given below) that if there exists a morphism $f: M \rightarrow N^{\epsilon}$ with $\epsilon$-trivial kernel and cokernel, then $M$ and $N$ are $\epsilon$-interleaved. Hence, there is a close connection between interleavings and morphisms with kernels and cokernels of bounded size also in the multi-parameter landscape.

Lemma 12 For any injective $f: M \rightarrow N^{\epsilon}$ with $2 \epsilon$-trivial cokernel, there exists a morphism $g: N \rightarrow M^{\epsilon}$ such that $f$ and $g$ constitute an $\epsilon$-interleaving pair.

Proof We have the following commutative square for all $p \in \mathbb{R}^{2}$ :

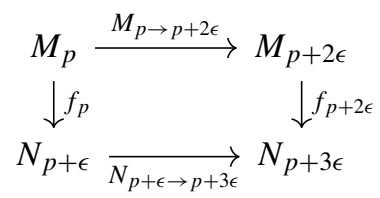

Let $n \in N_{p+\epsilon}$. Since $f$ has $2 \epsilon$-trivial cokernel and $f$ is injective, there exists a unique $m \in M_{p+2 \epsilon}$ such that $f_{p+2 \epsilon}(m)=N_{p+\epsilon \rightarrow p+3 \epsilon}(n)$. Define $g_{p}: N_{p+\epsilon} \rightarrow M_{p+2 \epsilon}$ by $g_{p}(n)=m$. Doing this for all $p \in \mathbb{R}^{2}$ defines a morphism $g: N^{\epsilon} \rightarrow M^{2 \epsilon}$ and we leave it to the reader to verify that $f$ and $g^{-\epsilon}$ define an $\epsilon$-interleaving pair.

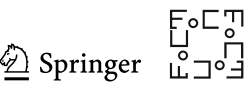


For fixed parameters $s, t \in[0, \infty]$, we consider the following computational problem:

$s$ - $t$-TRIVIAL- MORPHISM: Given two persistence modules $M, N$ in graded matrix representation, decide whether there exists a morphism $f: M \rightarrow N$ with $s$ trivial kernel and $t$-trivial cokernel.

Choosing $s=t=0$ simply asks whether the modules are isomorphic, which can be decided in polynomial time [6]. On the other extreme, $s=t=\infty$ imposes no conditions on the morphism, which turns the decision problem to be trivially true, using the 0-morphism. We show

Theorem $6 s$-t $t$-TRIVIAL- MORPHISM is NP-completefor every $(s, t) \notin\{(0,0),(\infty, \infty)\}$.

The case $(s, t)$ is computationally equivalent to the case $(c s, c t)$ with $c>0$, since we can scale all grades occurring in $M$ and $N$ by a factor of $c$. So, it suffices to prove hardness of 2- $t$-TRIVIAL- MORPHISM, $s$-2-TRIVIAL- MORPHISM (we will see that the choice of 2 will be convenient in the argument), $\infty$-0-TRIVIAL-MORPHISM and 0 - $\infty$-TRIVIAL- MORPHISM.

Note that for any choice of $s$ and $t, s$ - $t$-TRIVIAL- MORPHISM is in NP. The argument is similar to the first part of the proof of Theorem 3: a morphism can be specified in polynomial size with respect to the module sizes, and we can check the triviality conditions of the kernel and cokernel by considering ranks of matrices.

For the hardness, we first focus on the case $(s, 2)$; hence, we want to decide the existence of a morphism with $s$-trivial kernel and 2-trivial cokernel. The following simple observation is the key insight of the proof.

Lemma 13 Let $M, N$ be as in Theorem 2. Any morphism $f: M \rightarrow N^{1}$ with 2-trivial cokernel is injective.

Proof Recall that both $M$ and $N$ are direct sums of $n$ staircase modules. Let $p$ be any point such that $\operatorname{dim} M_{p}=\operatorname{dim} N_{p}=n$, and observe that $M_{p \rightarrow q}=\mathrm{id}_{\mathbb{F}}, N_{p \rightarrow q}=\mathrm{id}_{\mathbb{F}}$ and $f_{p}=f_{q}$ for all $q \geq p$. In particular, if $q=p+(2,2)$, the induced map $\operatorname{coker}\left(f_{p}\right) \rightarrow \operatorname{coker}\left(f_{q}\right)$ is the identity, and since $f$ has a 2-trivial cokernel by assumption, the map is also the 0-map. Hence $\operatorname{coker}\left(f_{p}\right)$ is trivial, implying that the map $f_{p}$ is surjective, and hence also injective, and the same holds for $f_{q}$ with $q \geq p$.

Now consider $f_{r}$ for an arbitrary $r \in \mathbb{R}^{2}$. Let $q \geq r$ be a point satisfying $q \geq p$. Since the internal morphisms of $M$ are all injective and $f_{p}$ is injective, so is $f_{r}$.

In other words, for $M$ and $N^{1}$ as above, the answer to $s$-2-TRIVIAL- MORPHISM is independent of $s$. Moreover, it follows:

Corollary 2 With $M, N$ as above, there exists a morphism $f: M \rightarrow N^{1}$ with 2-trivial cokernel and s-trivial kernel if and only if $M$ and $N$ are 1-interleaved.

Proof If such a morphism exists, Lemma 13 guarantees that the morphism is in fact injective with 2 -trivial cokernel. Lemma 12 with $\epsilon=1$ guarantees that the modules are 1-interleaved. 
Vice versa, if $M$ and $N$ are 1-interleaved, there is a morphism $f$ with 2-trivial kernel and cokernel by Lemma 11. Again using Lemma 13 guarantees that $f$ is injective, hence has a 0-trivial kernel.

Corollary $3 s$-2-TRIVIAL- MORPHISM is NP-hard for all $s \in[0, \infty]$.

Proof Given a CI-instance, we transform it into modules $M$ and $N$ as in Sect. 4. Assuming a polynomial time algorithm for $s$-2-TRIVIAL- MORPHISM, we apply it on $\left(M, N^{1}\right)$. If the algorithm returns that a morphism exists, we know by Corollary 2 that $M$ and $N$ are 1-interleaved and therefore, the CI-instance has a solution. If no morphism exists, $M$ and $N$ are not 1-interleaved and therefore, the CI-instance has no solution. We can thus solve the CI problem in polynomial time.

\subsection{Dual Staircases}

We will prove that 2-t-TRIVIAL- MORPHISM is NP-hard by a reduction from $s$-2TRIVIAL- MORPHISM. First we need some notation. For a staircase $S$, let $S^{\circ}$ denote the interior of $S$, and for a staircase module $M_{l}$ supported on a staircase $S$, we let $M_{l}^{\circ}$ denote the interval module supported on $S^{\circ}$. Observe that there is a canonical injection $M_{l}^{\circ} \hookrightarrow M$ (given by $m \mapsto m$ ). It is also easy to see that $d_{s}(S, T)=d_{s}\left(S^{\circ}, T^{\circ}\right)$. Here $d_{s}$ for interiors of staircases is defined in the obvious way. The reason why we look at interiors is technical: We eventually end up with a dual module $\left(M^{\circ}\right)^{*}$, and taking interiors makes sure the changes in this dual module happen at given points instead of "immediately after" the points, which is needed for a graded matrix representation of the module.

Lemma 14 Let $M$ and $N$ be staircase decomposable modules. There exists an injection $f: M \rightarrow N$ with $\epsilon$-trivial cokernel if and only if there exists an injection $f^{\circ}: M^{\circ} \rightarrow$ $N^{\circ}$ with $\epsilon$-trivial cokernel.

Proof Let $M=\oplus_{i} M_{i}$ and $N=\oplus_{j} N_{j}$. Observe that $S \subseteq T$ if and only if $S^{\circ} \subseteq T^{\circ}$. Therefore, any morphism $M_{i}^{\circ} \rightarrow N_{j}^{\circ}$ extends to a morphism $M_{j} \rightarrow N_{j}$ in the obvious way. Conversely, any morphism $M \rightarrow N$ restricts to a morphism $M^{\circ} \rightarrow N^{\circ}$. It is not hard to see that extension and restriction are inverse functions. In particular, there is a one-to-one correspondence between morphisms $f: M \rightarrow N$ and $f^{\circ}: M^{\circ} \rightarrow N^{\circ}$.

Suppose $f^{\circ}$ is injective. For any point $p$, there exists a $\delta>0$ such that $M_{p \rightarrow p+\delta}$ and $N_{p \rightarrow p+\delta}$ are isomorphisms, which also gives $f_{p+\delta}^{\circ}=f_{p+\delta}$. Since $f^{\circ}$ is injective, $f_{p+\delta}^{\circ}=f_{p+\delta}$ is, and by using the isomorphisms, we get that $f_{p}$ is injective, too. Since $p$ was arbitrary, we conclude that $f$ is injective. The converse can be proved by using the dual fact that for any $p$, there exists a $\gamma$ such that $M_{p-\gamma \rightarrow p}^{\circ}$ and $N_{p-\gamma \rightarrow p}^{\circ}$ are isomorphisms.

Suppose that coker $f$ is not $\epsilon$-trivial, so there is a $p$ and an $m \in N_{p}$ such that $N_{p \rightarrow p+\epsilon}(m)$ is not in the image of $f_{p+\epsilon}$. Similarly to how we picked $\delta$ above, we can pick $\delta$ and $\gamma$ with $\delta \leq \gamma$ in a way that makes the following diagram commute, with

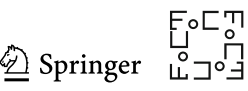


equalities and isomorphisms as shown.

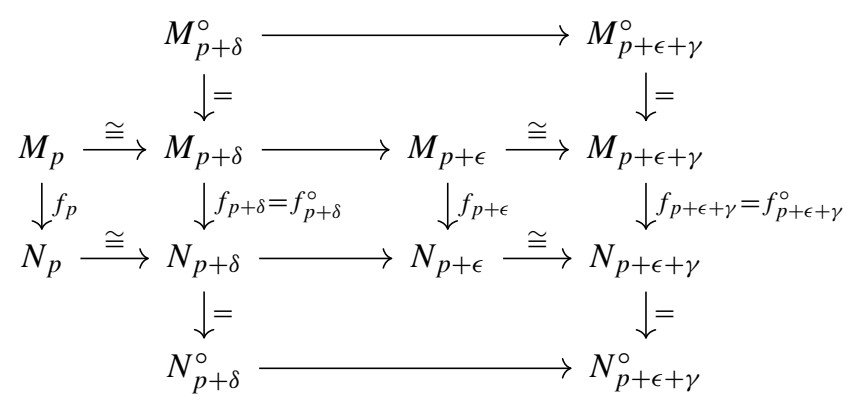

All the horizontal maps are internal morphisms. We know that $N_{p \rightarrow p+\epsilon}(m) \in N_{p+\epsilon}$ is not in the image of $f_{p+\epsilon}$. Let $m^{\prime} \in N_{p+\epsilon+\gamma}^{\circ}$ be the image of $m$ along the maps in the above diagram. Then $m^{\prime}$ is in the image of $N_{p+\delta \rightarrow p+\epsilon+\gamma}^{\circ}$, but not in the image of $f_{p+\epsilon+\gamma}^{\circ}$. Since $(\epsilon+\gamma)-\delta \geq \epsilon$, this shows that $f^{\circ}$ is not $\epsilon$-trivial. Again, the argument can be dualized to show the converse.

For an interval $I \subseteq \mathbb{R}^{2}$, define the dual interval $I^{*}$ as follows: $(x, y) \in I^{*}$ if and only if $(-x,-y) \in I$. And for an interval module $M_{l}$ supported on $I$, let $M_{l}^{*}$ denote be the interval module supported on $I^{*}$. If $M=\oplus_{i} M_{i}$ is a sum of interval modules $M_{i}$, then $M^{*}=\oplus_{i}\left(M_{i}\right)^{*}$. This is equivalent to considering $M$ as a module indexed by $\mathbb{R}^{2}$ with the partial order reversed.

Let $M^{\circ}=\oplus_{i} M_{i}^{\circ}$ and $N^{\circ}=\oplus_{j} N_{j}^{\circ}$, where $M_{i}^{\circ}$ and $N_{j}^{\circ}$ are interval modules supported on interiors of staircases, and let $f^{\circ}: M^{\circ} \rightarrow N^{\circ}$. Observe that we can represent $f^{\circ}$ by a collection of matrices $\left\{A_{p}\right\}_{p \in \mathbb{R}^{2}}$, where $A_{p}$ is the matrix representation of $f_{p}^{\circ}$ with respect to the bases given by the non-trivial elements of $\left\{\left(M_{i}^{\circ}\right)_{p}\right\}_{i}$ and $\left\{\left(N_{j}^{\circ}\right)_{p}\right\}_{j}$. Similarly, for any $p \leq q$, we can represent the linear maps $M_{p \rightarrow q}^{\circ}$ and $N_{p \rightarrow q}^{\circ}$ by matrices with respect to the obvious bases.

Importantly, representing $f_{p}^{\circ}$ by matrices $A_{p}$ as above, we get a dual morphism $\left(f^{\circ}\right)^{*}: N^{*} \rightarrow M^{*}$ given by the matrices $\left\{\left(A_{-p}\right)^{T}\right\}_{p \in \mathbb{R}^{2}}$. This induces a bijection between the set of morphisms from $M^{\circ}$ to $N^{\circ}$ and the set of morphisms from $\left(N^{\circ}\right)^{*}$ to $\left(M^{\circ}\right)^{*}$.

Lemma $15 f^{\circ}$ is an injection with $\epsilon$-trivial cokernel if and only if $\left(f^{\circ}\right)^{*}$ is a surjection with $\epsilon$-trivial kernel.

Proof The first part is straightforward: the matrix $A_{p}$ represents a surjective linear map if and only if $A_{p}^{T}$ represents an injective linear map. Since $\left(f^{\circ}\right)_{p}^{*}=f_{-p}^{\circ}$, the result follows readily.

For the second part, let $p$ be any point in $\mathbb{R}^{2}$, and let $X$ be the matrix representation of the morphism $N_{p \rightarrow p+\epsilon}^{\circ}$ with respect to the basis given by the $N_{j}^{\circ}$ 's. Then, by construction, $X^{T}$ is a matrix representation for $\left(N^{\circ}\right)_{-p-\epsilon \rightarrow-p}^{*}$ (with respect to the dual bases $)$. Using the elementary fact that $\operatorname{col}(X) \subseteq \operatorname{col}\left(A_{p+\epsilon}\right)$ if and only if $\operatorname{ker}\left(A_{p+\epsilon}^{T}\right) \subseteq \operatorname{ker}\left(X^{T}\right)$, where $\operatorname{col}(X)$ denotes the column space of $X$, we conclude 


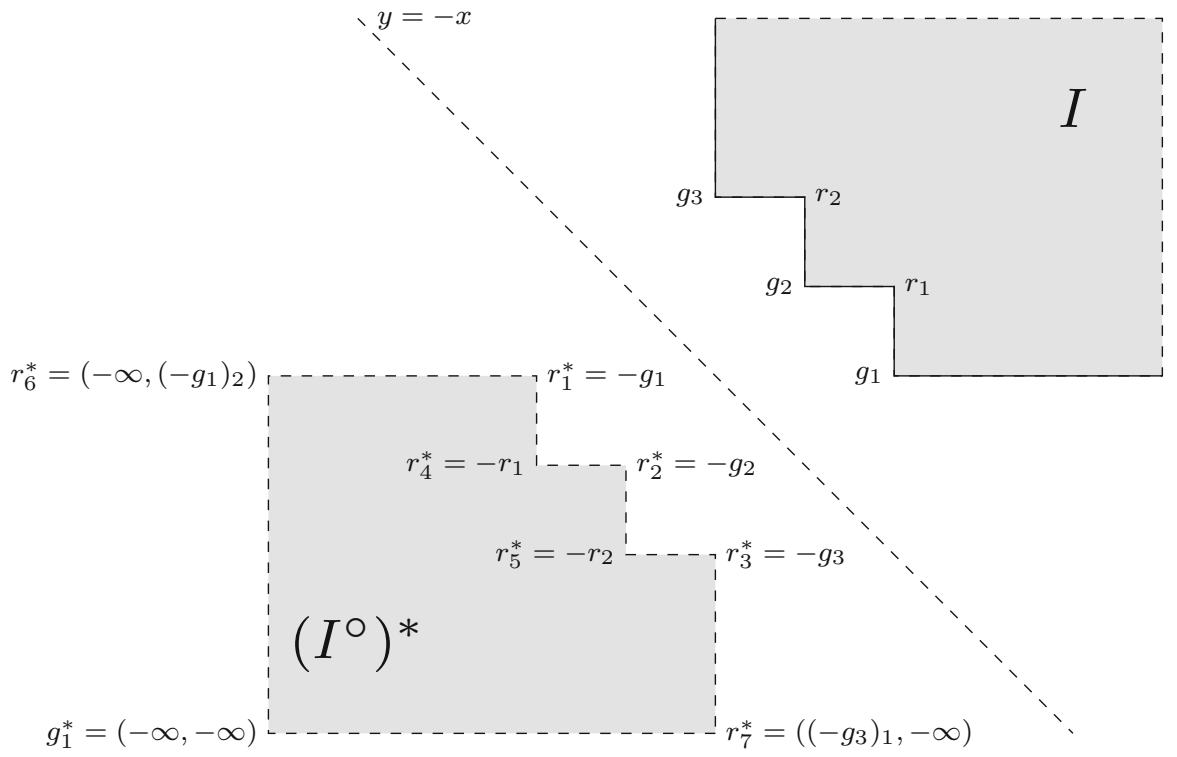

Fig. 5 The staircase module $k_{I}$ supported on the interval $I$ admits a graded matrix representation with $G=$

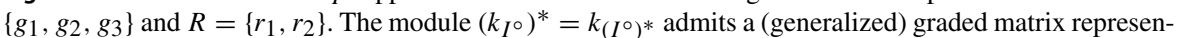
tation with $G^{*}=\{(-\infty,-\infty)\}$ and $R^{*}=\left\{-g_{1},-g_{2},-g_{3},-r_{1},-r_{2},\left(-\infty,\left(-g_{1}\right)_{2}\right),\left(\left(-g_{3}\right)_{1},-\infty\right)\right\}$. In the proof of Corollary 4 , we may replace $\infty$ with $z \gg 0$ to obtain a proper graded matrix representation

that $\operatorname{im}\left(N_{p \rightarrow p+\epsilon}^{\circ}\right) \subseteq \operatorname{im}\left(f_{p+\epsilon}^{\circ}\right)$ if and only if $\operatorname{ker}\left(\left(f^{\circ}\right)_{-p-\epsilon}^{*}\right) \subseteq \operatorname{ker}\left(\left(N^{\circ}\right)_{-p-\epsilon \rightarrow p}^{*}\right)$. As $p$ was arbitrary, this concludes the proof.

Corollary 4 2-t-TRIVIAL-MORPHISM is NP-hard for all $t \in[0, \infty]$.

Proof This follows from the previous two lemmas and Corollary 3 . There is however a technical obstacle arising from the fact that $\left(M^{\circ}\right)^{*}$ and $\left(N^{\circ}\right)^{*}$ have their generators at grade $(-\infty,-\infty)$. This problem is easy to solve, either by altering the graded matrix representation to allow such a generator, or by placing all generators at a sufficiently small value $p \in \mathbb{R}^{2}$ that is smaller than all corners of the staircase, see Fig. 5 for an illustration. Introducing such a minimal grade does not invalidate any of the given arguments—we omit the technical details.

\subsection{Surjective Morphisms}

After Corollaries 3 and 4, all we have left to prove Theorem 6 is the cases $\infty-0$ TRIVIAL- MORPHISM and $0-\infty$-TRIVIAL- MORPHISM. Recall that these correspond to asking for a surjection in the first case and an injection in the second.

Lemma $16 \infty$-0-TRIVIAL- MORPHISM and 0- $\infty$-TRIVIAL- MORPHISM are both NPhard. 
Proof We will only prove the first case; the second follows by dualizing the arguments in an appropriate way, for instance by using dual staircases as above.

Recall that we have assumed $\mathbb{F}$ to be finite. Let $q$ denote the number of elements in $\mathbb{F}$, and assume that $\phi$ is a $3 \mathrm{CNF}$ formula with $n$ variables $\left\{x_{1}, \ldots, x_{n}\right\}$ and $m$ clauses $\left\{c_{1}, \ldots, c_{m}\right\}$. We shall construct modules $M=A \oplus B \oplus\left(\oplus_{i=1}^{n} \oplus_{r=1}^{q} M_{i}^{r}\right)$ and $N=N_{1} \oplus N_{2}$, where $M_{i}^{r}, A, B, N_{1}$ and $N_{2}$ are staircase modules, in such a way that there exists a surjection $M \rightarrow N$ if and only if $\phi$ is satisfiable. Importantly, we know from Lemma 7 that any morphism between staircase decomposable modules can be represented by a matrix with entries in $\mathbb{F}$. We only stated the result in the case where each module is built from the same number of staircases, but the same argument shows that a morphism $M \rightarrow N$ in this case is described by a $2 \times(n q+2)$-matrix, which we shall assume is ordered in the following way

$$
\left.\begin{array}{ccccccccc}
A & B & M_{1}^{1} & M_{1}^{2} & \ldots & M_{2}^{1} & \ldots & M_{n}^{q} & \\
* & * & * & * & \ldots & * & \ldots & * \\
* & * & * & * & \ldots & * & \ldots & *
\end{array}\right) N_{1}
$$

Furthermore, recall that any staircase module is defined by a set of generators, i.e., a set of incomparable points defining the "corners" of the staircase. It is not hard to see that a morphism $M \rightarrow N$ is surjective if and only if it is surjective at the all the corners points of $N_{1}$ and $N_{2}$.

Let

$$
D=\left\{A, B, N_{1}, N_{2}\right\} \bigcup_{i, r}\left\{M_{i}^{r}\right\}
$$

and let $S \subseteq \mathbb{R}^{2}$ be a set of pairwise incomparable points. Any function $G: S \rightarrow P(D)$, where $P(D)$ is the power set of $D$, specifies the modules in the decomposition of $M$ and $N$ by enforcing that $X \in D$ has a corner point at $s \in S$ if and only if $X \in G(s)$. In what follows we shall define such a function $G$ in four steps, and define the staircase modules in $D$ accordingly.

Let $S=\left\{a, b, g_{i}^{r}, g_{i}^{r, s}, h_{j}^{y, z, w}\right\}$ be a set of distinct incomparable points in $\mathbb{R}^{2}$, where $i, j, r, s, y, z, w$ run through indices which will be defined as we define $G$. In the initial step, we define $G(a)=\left\{A, N_{1}\right\}$ and $G(b)=\left\{B, N_{2}\right\}$. The addition of these corners enforce that the matrix (in the ordering given above) must be of the form

$$
\left(\begin{array}{lllll}
1 & 0 & * & \ldots & * \\
0 & 1 & * & \ldots & *
\end{array}\right)
$$

This can be seen as follows: since $a$ and $b$ are incomparable, and $a \in A$ while $a \notin N_{2}$, we must have that $N_{2} \nsubseteq A$. Lemma 6 allows us to conclude that the only morphism from $A \rightarrow N_{2}$ is the trivial one. Similarly we see that the morphism $B \rightarrow N_{1}$ must be the trivial one. Furthermore, since $M_{a}=A_{a}$ and $N_{a}=\left(N_{2}\right)_{a}$, surjectivity at $a$ implies that $A \rightarrow N_{1}$ must be non-zero, which gives the non-zero entry in the first column. We can multiply any column in the matrix with a non-zero element without changing 
the validity or surjectivity of the morphism, so we can assume that this element is 1 . Similarly we get a 1 in the second row of the second column.

We proceed our inductive step by defining $G\left(g_{i}^{r}\right)=\left\{A, M_{i}^{r}, N_{1}, N_{2}\right\}$ for all $1 \leq$ $i \leq n$ and $1 \leq r \leq q$. Restricting the matrix to the columns corresponding to $A$ and $M_{i}^{r}$ we get

$$
\left(\begin{array}{ll}
1 & * \\
0 & *
\end{array}\right)
$$

For the morphism to be surjective at the point $g_{i}^{r}$, this matrix must be of full rank. Therefore, we can write it as

$$
\left(\begin{array}{cc}
1 & d_{i}^{r} \\
0 & 1
\end{array}\right)
$$

where we again have used the fact that we can scale columns by non-zero constants. In other words, any surjection $M \rightarrow N$ must be of the form

$$
\left(\begin{array}{cccccccccccc}
1 & 0 & d_{1}^{1} & \ldots & d_{1}^{q} & d_{2}^{1} & \ldots & d_{2}^{q} & \ldots & d_{n}^{1} & \ldots & d_{n}^{q} \\
0 & 1 & 1 & \ldots & 1 & 1 & \ldots & 1 & \ldots & 1 & \ldots & 1
\end{array}\right)
$$

Continuing, let $G\left(g_{i}^{r, s}\right)=\left\{M_{i}^{r}, M_{i}^{s}, N_{1}, N_{2}\right\}$, for all $1 \leq i \leq n$ and $1 \leq r<s \leq q$. Restricting the matrix to the columns corresponding to $M_{i}^{r}$ and $M_{i}^{s}$ yields the matrix

$$
\left(\begin{array}{cc}
d_{i}^{r} & d_{i}^{s} \\
1 & 1
\end{array}\right)
$$

For the matrix to be surjective at $g_{i}^{r, s}$, also this matrix must be of full rank. In particular, it must be the case that $d_{i}^{r} \neq d_{i}^{s}$, and therefore exactly one of $d_{i}^{1}, \ldots, d_{i}^{q}$ equals 0 . We will interpret $d_{i}^{1}=0$ as choosing $x_{i}$ to be false, and $d_{i}^{1} \neq 0$ as choosing $x_{i}$ to be true.

What remains is to encode the clauses of $\phi$. For a clause $c_{j}$, let $x_{\alpha_{j, 1}}, x_{\alpha_{j, 2}}, x_{\alpha_{j, 3}}$ be the variables such that either the variable itself or its negation occurs in $c_{j}$, with $\alpha_{j, 1}<\alpha_{j, 2}<\alpha_{j, 3}$. For $1 \leq i \leq 3$, let $X_{j}^{i}=\{1\}$ if $x_{\alpha_{j, i}}$ occurs in $c_{j}$; if instead its negation occurs, let $X_{j}^{i}=\{2, \ldots, q\}$. For example, if $c_{j}=x_{1} \vee \neg x_{2} \vee \neg x_{4}$, then $\alpha_{j, 1}=1, \alpha_{j, 2}=2$ and $\alpha_{j, 3}=4$, and $X_{j}^{1}=\{1\}, X_{j}^{2}=\{2, \ldots, q\}$ and $X_{j}^{3}=$ $\{2, \ldots, q\}$. Define $G\left(h_{j}^{y, z, w}\right)=\left\{B, M_{\alpha_{j, 1}}^{r}, M_{\alpha_{j, 2}}^{s}, M_{\alpha_{j, 3}}^{t}, N_{1}, N_{2}\right\}$, for all $1 \leq j \leq m$ and $y \in X_{j}^{1}, z \in X_{j}^{2}, w \in X_{j}^{3}$.

This time, the following submatrix must have rank 2 for all $h_{j}^{y, z, w}$ with $j, y, z, w$ as above.

$$
\left(\begin{array}{cccc}
0 & d_{\alpha_{j, 1}}^{y} & d_{\alpha_{j, 2}}^{z} & d_{\alpha_{j, 3}}^{w} \\
1 & 1 & 1 & 1
\end{array}\right)
$$

At this stage, we have concluded the construction of the modules and no further restrictions will be imposed on the matrix. In particular, the above shows that there

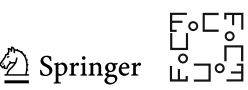


exists a surjection $M \rightarrow N$ if and only if there is an assignment $d_{i}^{r} \in \mathbb{F}$ such that the following is satisfied:

$-\mathbb{F}=\left\{d_{i}^{1}, \ldots, d_{i}^{q}\right\}$ for all $1 \leq i \leq n$.

- The matrix of (5) has full rank for every $h_{j}^{y, z, w}$.

We show that this is equivalent to $\phi$ being satisfiable.

" $\Rightarrow$ ": Assume that $\phi$ is satisfiable and pick a satisfying assignment. If $x_{i}$ is set to false, then define $d_{i}^{1}=0$. If $x_{i}$ is set to true, then define $d_{i}^{2}=0$. In both cases, we assign the remaining variables values such that $\mathbb{F}=\left\{d_{i}^{1}, \ldots, d_{i}^{q}\right\}$ for all $1 \leq i \leq n$. Consider the clause $c_{j}$ as above, and assume that $x_{\alpha_{j, l}}$ is assigned a truth value such that the literal associated to $x_{\alpha_{j, l}}$ in $c_{j}$ evaluates to true. Then $d_{\alpha_{j, l}}^{y} \neq 0$ for all $y \in X_{j}^{l}$, implying that the matrix of (5) has rank 2 for all $h_{j}^{y, z, w}$.

" $\Leftarrow$ ": Assume an assignment of the variables $d_{i}^{r}$ satisfying the two bullet points above, and set $x_{i}$ to be false if $d_{i}^{1}=0$, and true otherwise. Consider the clause $c_{j}$ as above, and observe that there exists an index $y \in X_{j}^{l}$ such that $d_{\alpha_{j, l}}^{y}=0$ if and only if the literal in $c_{j}$ associated to $x_{\alpha_{j, l}}$ evaluates to false. In particular, $c_{j}$ evaluates to true if and only if at least one of $d_{\alpha_{j, 1}}^{y}, d_{\alpha_{j, 2}}^{z}$ and $d_{\alpha_{j, 3}}^{w}$ is non-zero for every $(x, y, z) \in X_{j}^{1} \times X_{j}^{2} \times X_{j}^{3}$. This is equivalent to the matrix of (5) having full rank for every $h_{j}^{y, z, w}$.

In the end, we have a reduction from 3SAT to $\infty$-0-TRIVIAL- MORPHISM. To complete the proof, we must show that the instance of $\infty$-0-TRIVIAL- MORPHISM can be constructed in polynomial time in the input size of the instance of 3SAT. As we have assumed $q$ to be fixed and finite, it suffices to observe that $M$ is defined by $n q+2$ staircase modules, while $N$ is a sum of 2 staircase modules, and that each of these are generated by at most $2+n q+n\left(\frac{q}{2}\right)+m(q-1)^{3}$ generators. We remark that the generators can be chosen along the antidiagonal $x=-y$ in $\mathbb{R}^{2}$.

An interesting point is that the number of generators of the staircases in the proof increases with the size of $\mathbb{F}$. Hence, the proof strategy only applies in the setting of a finite field (with a constant number of elements).

We conclude this section by remarking that $0-\infty$-TRIVIAL- MORPHISM is equivalent to the problem of deciding if a module $M^{\prime}$ is a submodule of another persistence module $M$. Interestingly, it can be checked in polynomial time if $M^{\prime}$ is a summand of $M[6$, Theorem 3.5].

\section{A Distance Induced by a Noise System}

As a last application of our methods, we show that a particular distance induced by a noise system is NP-hard to approximate within a factor of 2.

A noise system, as introduced by Scolamiero et al. [19], induces a pseudometric on (tame) persistence modules. In this section, we shall briefly consider one particular noise system and we refer the reader to [19] for an in-depth treatment of the more general theory. 
We say that $f: M \rightarrow N$ is a $\mu$-equivalence if $f$ has $\mu_{1}$-trivial kernel and $\mu_{2}$-trivial cokernel, and $\mu_{1}+\mu_{2} \leq \mu$. From this definition, we can define the following distance between two persistence modules $M$ and $N$

$d_{\text {noise }}(M, N)$

$=\inf \{\mu \mid \exists M \stackrel{f}{\leftarrow} X \stackrel{g}{\rightarrow} N, f$ an $\epsilon$-equivalence, $g$ a $\delta$-equivalence and $\epsilon+\delta \leq \mu\}$

The reader may verify that this distance coincides with the distance induced by the noise system $\left\{S_{\epsilon}\right\}$ where $S_{\epsilon}$ consists of all persistence modules $M$ with the property that $M_{p \rightarrow p+(\epsilon, \epsilon)}$ is trivial for all $p$. In particular, $d_{\text {noise }}$ is indeed an extended pseudometric [19, Proposition 8.7].

Like for the interleaving distance, we can define the computational problem of $c$-approximating $d$ for a constant $c \geq 1$.

$c$-APPROX- $d_{\text {noise }}$ : Given two persistence modules $M, N$ in graded matrix representation, return a real number $r$ such that

$$
d_{\text {noise }}(M, N) \leq r \leq c \cdot d_{\text {noise }}(M, N)
$$

Theorem 7 -APPROX-d is NP-hard for $c<2$.

Proof Let $(n, P, Q)$ be a CI-instance and construct $M$ and $N$ as in Theorem 2 . We will show the following implications:

$$
\begin{aligned}
& d_{I}(M, N)=1 \Rightarrow d_{\text {noise }}\left(M, N^{1}\right) \leq 2 \\
& d_{I}(M, N)=3 \Rightarrow d_{\text {noise }}\left(M, N^{1}\right) \geq 4 .
\end{aligned}
$$

This allows us to conclude that an algorithm $c$-approximating $d_{\text {noise }}(M, N)$ for $c<2$ will return a number $<4$ if $d_{I}(M, N)=1$ and a number $\geq 4$ if $d_{I}(M, N)=3$. This constitutes a polynomial time reduction from CI to 2-APPROX- $d_{\text {noise }}$ and the result follows from Theorem 1 .

First assume that $d_{I}(M, N)=1$. Let $X=M$ with $f: X \rightarrow M$ the identity morphism. Lemma 11 shows that the interleaving morphism $g: M \rightarrow N^{1}$ has 2-trivial cokernel, and from Lemma 13 we know that it is injective. Hence $g$ is a 2-equivalence and thus $d_{\text {noise }}\left(M, N^{1}\right) \leq 2$.

Now assume that $d_{\text {noise }}\left(M, N^{1}\right)<4$. By definition this gives a diagram $M \stackrel{f}{\leftarrow}$ $X \stackrel{g}{\rightarrow} N^{1}$ where $f$ is an $\epsilon$-equivalence, $g$ is a $\delta$-equivalence, and $\epsilon+\delta<4$. We may assume that both $f$ and $g$ are injective. To see this, consider $x \in \operatorname{ker}\left(f_{p}\right)$. Because $f$ is an $\epsilon$-equivalence, $\operatorname{ker}(f)$ is $\epsilon$-trivial, so $X_{p \rightarrow p+(\epsilon, \epsilon)}(x)=0$. This gives

$$
0=g_{p+(\epsilon, \epsilon)} \circ X_{p \rightarrow p+(\epsilon, \epsilon)}(x)=N_{p \rightarrow p+(\epsilon, \epsilon)}^{1} \circ g_{p}(x) .
$$

Since $N_{p \rightarrow p+(\epsilon, \epsilon)}^{1}$ is injective, we conclude that $g_{p}(x)=0$. This shows that $\operatorname{ker}(f) \subseteq$ $\operatorname{ker}(g)$, and by symmetry, that $\operatorname{ker}(f)=\operatorname{ker}(g)$. Replacing $X$ with $\tilde{X}=X / \operatorname{ker}(f)$

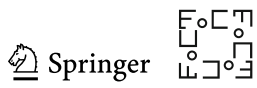


induces injective morphisms $M \stackrel{\tilde{f}}{\leftarrow} \tilde{X} \stackrel{\tilde{g}}{\rightarrow} N^{1}$ with the properties that $\tilde{f}$ is an $\epsilon$ equivalence and that $\tilde{g}$ is a $\delta$-equivalence. Hence $f$ and $g$ may be assumed to be injective. Under this assumption, we get the following two inequalities from Lemma 12

$$
\begin{array}{r}
d_{I}\left(M, X^{\epsilon / 2}\right) \leq \epsilon / 2 \\
d_{I}\left(N^{1}, X^{\delta / 2}\right) \leq \delta / 2 .
\end{array}
$$

Observe that $d_{I}\left(N^{1}, X^{\delta / 2}\right)=d_{I}\left(N^{1-\delta / 2}, X\right)=d_{I}\left(N^{1+(\epsilon-\delta) / 2}, X^{\epsilon / 2}\right)$. Together with the first inequality, this gives $d_{I}\left(M, N^{1+(\epsilon-\delta) / 2}\right) \leq(\epsilon+\delta) / 2$, and thus

$$
\begin{aligned}
d_{I}(M, N) & \leq d_{I}\left(M, N^{1+(\epsilon-\delta) / 2}\right)+d_{I}\left(N^{1+(\epsilon-\delta) / 2}, N\right) \\
& \leq(\epsilon+\delta) / 2+(1+(\epsilon-\delta) / 2)=1+\epsilon .
\end{aligned}
$$

To conclude the proof, we will show that $\delta \geq 2$, as this implies $1+\epsilon<1+4-\delta \leq 3$. Assuming that $n \geq 1$, let $p$ be such that $\operatorname{dim} N_{p}>0$ and $\operatorname{dim} M_{p+(r, r)}=0$ for all $r<1$. Such a point exists for the following reason: let $M_{i}$ be any indecomposable summand of $M$ and let $N_{j}$ be any indecomposable summand of $N$. Then $M_{i}$ is a staircase module for which the underlying staircase is obtained by moving certain corners of the staircase $S$ in Fig. 2. Likewise, the staircase supporting $N_{j}$ is obtained by moving certain corners of $T$. However, by construction, and as shown in Fig. 3, a number of corners are left unmoved. Hence, we may simply choose $p$ to be any corner point of $T$ with negative 1st coordinate which is left unmoved in the construction of $N_{j}$.

Let $q=p-(1,1)$. Then $\operatorname{dim} N_{q}^{1}>0$ and $\operatorname{dim} M_{q+(r, r)}=0$ for all $r<2$. But since $f$ is an injection, the space $X_{q+(r, r)}$ must also be trivial for any $r<2$. It follows that $\delta \geq 2$.

\section{Conclusion}

Using the link between persistence modules indexed over $\mathbb{R}^{2}$ and CI problems introduced in [4], we settle the computational complexity of a series of problems. Most notably, we show that computing the interleaving distance is NP-hard, as is approximating it to any constant factor $<3$. Moreover, we investigated the problem of deciding one-sided stability. Except for checking isomorphism, which is known to be polynomial, we show that all non-trivial cases are NP-hard. This includes checking whether a module is a submodule of another. Our assumption that we are working over a finite field stays in the background for most of the paper, but we rely heavily on this assumption for proving the submodule problem. Lastly, we showed that approximating a distance $d$ arising from a noise system up to a constant less than 2 is also NP-hard.

Throughout, we use persistence modules decomposing into very simple modules called staircase modules. These have the big advantage that the morphisms between them have very simple descriptions in terms of matrices. While this simplification

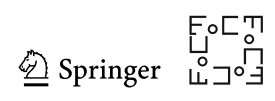


might appear to throw the complexity out with the bathwater, our results clearly show that this is not the case.

The question of whether $c$-APPROX-INTERLEAVING- DISTANCE is NP-hard for $c \geq 3$ is still open, and it is not clear whether one can prove this with CI problems or not. Even if this should not be possible, we believe that a better understanding of CI problems would lead to a better understanding of persistence modules and interleavings.

Acknowledgements We thank the anonymous referees for valuable suggestions, including the connection to noise systems discussed in Sect. 7. Magnus Bakke Botnan has been partially supported by the DFG Collaborative Research Center SFB/TR 109 "Discretization in Geometry and Dynamics". Michael Kerber is supported by Austrian Science Fund (FWF) Grant Number P 29984-N35.

Open Access This article is distributed under the terms of the Creative Commons Attribution 4.0 International License (http://creativecommons.org/licenses/by/4.0/), which permits unrestricted use, distribution, and reproduction in any medium, provided you give appropriate credit to the original author(s) and the source, provide a link to the Creative Commons license, and indicate if changes were made.

\section{References}

1. Bauer, U., Lesnick, M.: Induced matchings of barcodes and the algebraic stability of persistence. In: Proceedings of the thirtieth annual symposium on Computational geometry, p. 355. ACM (2014)

2. Bendich, P., Marron, J.S., Miller, E., Pieloch, A., Skwerer, S.: Persistent homology analysis of brain artery trees. The annals of applied statistics 10 1, 198-218 (2016)

3. Bjerkevik, H.B.: Stability of higher-dimensional interval decomposable persistence modules. arXiv preprint arXiv:1609.02086 (2016)

4. Bjerkevik, H.B., Botnan, M.B.: Computational complexity of the interleaving distance. In: 34 th International Symposium on Computational Geometry, SoCG 2018, pp. 13:1-13:15 (2018)

5. Botnan, M.B., Lesnick, M.: Algebraic stability of zigzag persistence modules. Algebraic \& Geometric Topology 18, 3133-3204 (2018)

6. Brooksbank, P.A., Luks, E.M.: Testing isomorphism of modules. Journal of Algebra 320(11), 40204029 (2008)

7. Buchet, M., Escolar, E.G.: Realizations of indecomposable persistence modules of arbitrarily large dimension. In: 34th International Symposium on Computational Geometry, SoCG 2018, pp. 15:1$15: 13$ (2018)

8. Chazal, F., De Silva, V., Glisse, M., Oudot, S.: The structure and stability of persistence modules. Springer, New York (2016)

9. Chazal, F., Guibas, L.J., Oudot, S.Y., Skraba, P.: Persistence-based clustering in riemannian manifolds. J. ACM 60(6), 41:1-41:38 (2013)

10. Dey, T.K., Xin, C.: Computing bottleneck distance for 2-d interval decomposable modules. In: 34th International Symposium on Computational Geometry, SoCG 2018, pp. 32:1-32:15 (2018)

11. Edelsbrunner, H., Harer, J.: Computational Topology: An Introduction. American Mathematical Society, Providence, RI, USA (2010)

12. Ivanyos, G., Karpinski, M., Saxena, N.: Deterministic polynomial time algorithms for matrix completion problems. SIAM journal on computing 39(8), 3736-3751 (2010)

13. Kerber, M., Nigmetov, A.: Geometry helps to compare persistence diagrams. ACM Journal of Experimental Algorithmics 22 (2017)

14. Lesnick, M.: The theory of the interleaving distance on multidimensional persistence modules. Foundations of Computational Mathematics 15(3), 613-650 (2015)

15. Lesnick, M., Wright, M.: Interactive visualization of 2-d persistence modules. arXiv preprint arXiv:1512.00180 (2015)

16. Pranav, P., Edelsbrunner, H., van de Weygaert, R., Vegter, G., Kerber, M., Jones, B., Wintraecken, M.: The topology of the cosmic web in terms of persistent betti numbers. Monthly Notices of the Royal Astronomical Society 465(4), 4281-4310 (2016)

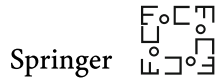


17. Reininghaus, J., Huber, S., Bauer, U., Kwitt, R.: A stable multi-scale kernel for topological machine learning. In: IEEE Conference on Computer Vision and Pattern Recognition, CVPR 2015, Boston, MA, USA, June 7-12, 2015, pp. 4741-4748 (2015)

18. Rybakken, E., Baas, N., Dunn, B.: Decoding of neural data using cohomological feature extraction. Neural computation 31(1), 68-93 (2019)

19. Scolamiero, M., Chachólski, W., Lundman, A., Ramanujam, R., Öberg, S.: Multidimensional persistence and noise. Foundations of Computational Mathematics 17(6), 1367-1406 (2017)

Publisher's Note Springer Nature remains neutral with regard to jurisdictional claims in published maps and institutional affiliations. 